\title{
Quandles of cyclic type with several fixed points
}

\author{
António Lages Pedro Lopes* \\ Department of Mathematics \\ Instituto Superior Técnico \\ Universidade de Lisboa \\ Av. Rovisco Pais, 1049-001 Lisbon, Portugal \\ \{antonio.lages, pedro.f.lopes\}@tecnico.ulisboa.pt
}

Submitted: Mar 2, 2019; Accepted: Aug 15, 2019; Published: Aug 30, 2019

(C) The authors. Released under the CC BY-ND license (International 4.0).

\begin{abstract}
A quandle of cyclic type of order $n$ with $f$ (greater than 1) fixed points is such that, by definition, each of its permutations splits into $f$ cycles of length 1 and one cycle of length $n-f$. In this article we prove that there is only one such connected quandle, up to isomorphism. This is a quandle of order 6 and 2 fixed points, known in the literature as octahedron quandle.
\end{abstract}

Mathematics Subject Classifications: $20 \mathrm{~N} 02$

\section{Introduction}

\subsection{Quandles}

The algebraic structure known as quandle appeared first in the literature in 1982, due to Joyce [11] and Matveev [15], independently (see also [8] and [9]). It was designed to constitute the algebraic counterpart to the Reidemeister moves [13]. As such it turned out to be an important tool in telling knots apart [7], [2], [5]. Algebraists also find it interesting in the domain of Hopf algebras [1]. It thus seem relevant to study the structure of quandles. In the current article we take another step in this direction by investigating and almost fully classifying a family of quandles. In [14] quandles are regarded as sequences of permutations and based on the features of permutations conclusions are drawn. In particular, in [14] quandles of the following sort are looked into. Given a positive integer $n$ we consider a quandle of order $n$, each of whose permutations split into a cycle of length $n-1$ and a fixed point; this fixed point complies with one of the quandles axioms - this is all detailed below. These quandles were subsequently called "quandles of cyclic type".

\footnotetext{
*Supported by project FCT PTDC/MAT-PUR/31089/2017, "Higher Structures and Applications".
} 
They were also studied in [12] and [17]. In this article, we work in the spirit of [14] i.e., quandles as sequences of permutations (see also [10] and [18], [19], [20]), and we look into the classification of a generalization of "quandles of cyclic type" which we call "quandles of cyclic type with several fixed points" (details are supplied below in the text). As a matter of fact, our considerations stem from a certain quandle of order six with two fixed points. We wondered about quandles whose permutations split into one long cycle and $f$ fixed points $(f \geqslant 2)$. It turns out that for infinitely many values of the parameters order, $n$, and number of fixed points, $f$, namely $n>2 f$, there is only one such quandle: our quandle of order six and two fixed points - this is proved in this article. This quandle was first referred to in [4] where it is described as the conjugacy class of the six cycles of length four from an alphabet with four letters, and used as an example in calculations of quandle homology. Then it appears in [1] (page 188) as the symmetries of a regular octahedron under rotations about an axis containing opposite vertices, see Figure 1 (the underlying set is the set of vertices).

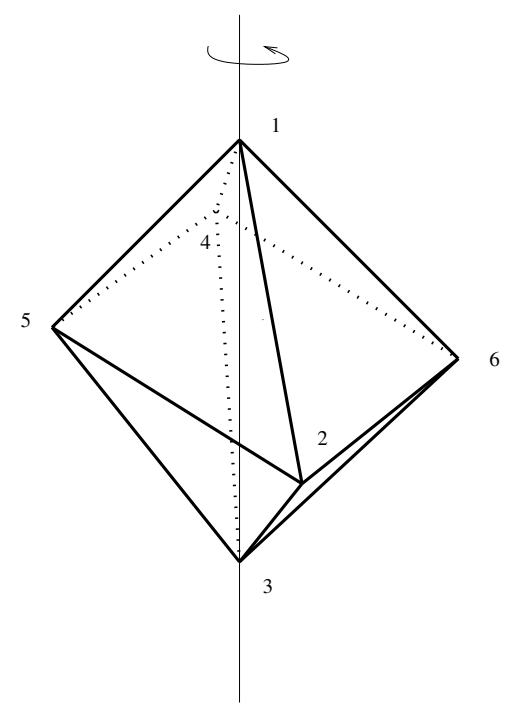

Figure 1: Geometric materialization of the "Octahedron Quandle". Numbering of vertices is consistent with Table 1, the "multiplication" table for this quandle. The axis depicted and the sense of rotation refer to the action of the first quandle element.

In [14], Roseman and the second author pick it as an example of a non-Alexander quandle, still proving to be a good quandle at telling knots apart via counting colorings. It was dubbed "Octahedron quandle".

\subsection{Basic Definitions and Results}

The algebraic structure known as quandle, introduced independently in [11] and [15], is defined as follows.

Definition 1. Let $Q$ be a set equipped with a binary operation denoted by $*$. The pair $(Q, *)$ is said to be a quandle if, for each $a, b, c \in Q$, 
1. $a * a=a$ (idempotency);

2. $\exists ! x \in Q: x * b=a$ (right-invertibility);

3. $(a * b) * c=(a * c) *(b * c)$ (self-distributivity).

We present a few examples of quandles.

\section{Example 2.}

- Let $G$ be a group and let $*$ be the binary operation on $G$ given by $a * b=b a b^{-1}$, for every $a, b \in G$, where the juxtaposition on the right-hand side denotes group multiplication. Then, the pair $(G, *)$ is a quandle;

- For each $n \geqslant 2,\left(R_{n}, *\right)$ denotes the quandle whose underlying set is $\mathbb{Z} / n \mathbb{Z}$ and whose operation is $a * b=2 b-a \bmod n$. This is called the dihedral quandle (of order $n$ );

- For each $n \geqslant 1,\left(T_{n}, *\right)$ denotes the quandle whose underlying set is $\{1, \ldots, n\}$ and whose operation is $i * j=i, \forall i, j \in\{1, \ldots, n\}$. This is called the trivial quandle (of order $n$ );

- $Q_{6}^{2}$ is the quandle whose multiplication table is displayed in Table 1.

\begin{tabular}{|l|llllll|}
\hline$*$ & 1 & 2 & 3 & 4 & 5 & 6 \\
\hline 1 & 1 & 5 & 1 & 6 & 4 & 2 \\
2 & 6 & 2 & 5 & 2 & 1 & 3 \\
3 & 3 & 6 & 3 & 5 & 2 & 4 \\
4 & 5 & 4 & 6 & 4 & 3 & 1 \\
5 & 2 & 3 & 4 & 1 & 5 & 5 \\
6 & 4 & 1 & 2 & 3 & 6 & 6 \\
\hline
\end{tabular}

Table 1: $Q_{6}^{2}$ multiplication table.

An alternative description of the structure of a quandle is the one given in the following theorem $([3],[9])$.

Theorem 3. Let $Q=\{1,2, \ldots, n\}$. Suppose a permutation $\mu_{i}$ from $S_{n}$, the symmetric group on $Q$, is assigned to each $i \in Q$. Then, the expression $j * i:=\mu_{i}(j), \forall j \in Q$, yields a quandle structure if and only if $\mu_{\mu_{i}(j)}=\mu_{i} \mu_{j} \mu_{i}^{-1}$ and $\mu_{i}(i)=i, \forall i, j \in Q$. This quandle structure is uniquely determined by the set of $n$ permutations, $\left\{\mu_{1}, \ldots, \mu_{n}\right\}$.

Proof. The proof can be found in [3].

In this article, we study the properties of each quandle by analyzing the structure uniquely determined by its set of $n$ permutations. We also only address finite quandles. If such a quandle has order $n$, we take the underlying set to be $\{1,2, \ldots, n\}$, without loss of generality. 
Definition 4. Given a quandle $(Q, *)$, its permutations are the $\mu_{i}$ 's referred to in the statement of Theorem 3, $\forall i \in Q$. Unless otherwise stated in the sequel, a $\mu_{i}$ always refers to a permutation from the quandle under discussion. We also write $(Q, \mu)$ to denote the same quandle from the point-of-view of permutations.

Definition 5. Let $(Q, *)$ (respect., $(Q, \mu))$ and $\left(Q^{\prime}, *^{\prime}\right)$ (respect., $\left.\left(Q^{\prime}, \mu^{\prime}\right)\right)$ be two quandles. A bijection $\alpha: Q \longrightarrow Q^{\prime}$ is a quandle isomorphism between these two quandles if, by definition, for any $i, j \in Q, \alpha(i * j)=\alpha(i) *^{\prime} \alpha(j)$.

Proposition 6. Keeping the notation and terminology of Defintion $4, \alpha$ is a quandle isomorphism if and only if, for any $i \in Q, \mu_{\alpha(i)}^{\prime}=\alpha \mu_{i} \alpha^{-1}$.

Proof. For any $i, j \in Q$,

$\alpha(j * i)=\alpha(j) *^{\prime} \alpha(i) \Longleftrightarrow \alpha\left(\mu_{i}(j)\right)=\mu_{\alpha(i)}^{\prime}(\alpha(j)) \Longleftrightarrow \alpha \mu_{i}=\mu_{\alpha(i)}^{\prime} \alpha \Longleftrightarrow \mu_{\alpha(i)}^{\prime}=\alpha \mu_{i} \alpha^{-1}$

\subsection{Definition of Quandle of Cyclic Type with Several Fixed Points}

As stated in Theorem 3, a quandle of order $n$ is uniquely determined by a set of $n$ permutations, where each one of these permutations can be decomposed into a set of disjoint cycles. The lengths of these cycles define the pattern of each permutation.

Definition 7. The pattern of a permutation is the list of the lengths of the disjoint cycles making up the permutation.

We can collect the information relative to the patterns of the $n$ permutations defining a quandle of order $n$ in order to define its profile.

Definition 8. The profile of a quandle of order $n$ is the list of the patterns of the $n$ permutations defining the quandle.

We now introduce the notion of connected quandle in order to state an important proposition.

Definition 9. A finite quandle $(Q, *)$ is said to be connected if,

$$
\begin{gathered}
\forall i, j \in Q, \exists k_{1}, k_{2}, \ldots, k_{n} \in Q: \\
j=\left(\cdots\left(\left(i * k_{1}\right) * k_{2}\right) * \cdots * k_{n}\right)=\mu_{k_{n}} \circ \cdots \circ \mu_{k_{2}} \circ \mu_{k_{1}}(i) .
\end{gathered}
$$

Proposition 10. Connected finite quandles have constant profiles.

Proof. Let $(Q, *)$ be a connected quandle. Then, given $i, j \in Q, \exists k_{1}, k_{2}, \ldots, k_{n} \in Q$ :

$j=\left(\cdots\left(\left(i * k_{1}\right) * k_{2}\right) * \cdots * k_{n}\right)=\mu_{k_{n}} \circ \cdots \circ \mu_{k_{2}} \circ \mu_{k_{1}}(i) \Rightarrow \mu_{j}=\mu_{k_{n}} \cdots \mu_{k_{2}} \mu_{k_{1}} \mu_{i} \mu_{k_{1}}^{-1} \mu_{k_{2}}^{-1} \cdots \mu_{k_{n}}^{-1}$,

by Theorem 3, and since conjugate permutations have the same pattern, the result follows. 
Remark 11. Note that quandles with constant profile do not have to be connected. For example, the trivial quandle of order $n,\left(T_{n}, *\right)$, has constant profile and it is not connected. The same for dihedral quandles of even order.

We now introduce the key notion of quandles of cyclic type with several fixed points.

Definition 12. Given $n, f \in \mathbb{N}, n-2 \geqslant f>1$, a quandle of cyclic type of order $n$ with $f$ fixed points is a quandle of order $n$ with constant profile given by

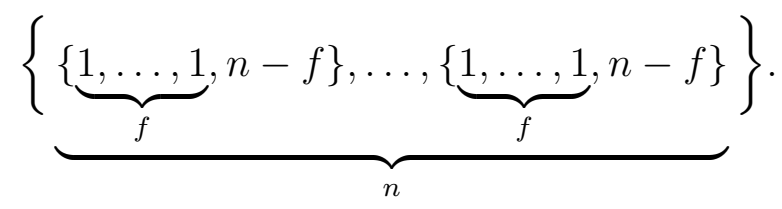

When there is no need to refer to its order or to its number of fixed points we refer to each of these quandles as quandle of cyclic type with several fixed points.

The previous definition means that each one of the $n$ permutations defining a quandle of cyclic type of order $n$ with $f$ fixed points splits into the following types and numbers of disjoint cycles. One cycle of length $n-f(>1)$ and $f$ cycles of length 1 .

In passing, we note, by inspection of Table 1 , that $Q_{6}^{2}$ is a connected quandle. Hence, by Proposition 10, it has a constant profile. But more than that, $Q_{6}^{2}$ is, in fact, a quandle of cyclic type of order 6 with 2 fixed points. In this article, we show that this is the only connected quandle of cyclic type with several fixed points.

In the sequel, we use the following notation.

Definition 13. Let $Q$ be a quandle of cyclic type with several fixed points of order $n$. As per Theorem 2.1.1, its $n$ permutations are denoted $\mu_{i}, i \in\{1, \ldots, n\}$. In particular, $\mu_{i}(i)=i, \forall i \in\{1, \ldots, n\}$. The set of fixed points of $\mu_{i}$ is denoted $F_{i}, i \in\{1, \ldots, n\}$. The set of points in the non-singular cycle of $\mu_{i}$ is denoted $C_{i}, i \in\{1, \ldots, n\}$. We note that $C_{i} \cap F_{i}=\emptyset$ and $C_{i} \cup F_{i}=\{1, \ldots, n\}, \forall i \in\{1, \ldots, n\}$.

\subsection{Statement of the Results in this Article}

Theorem 14. Let $n$ and $f$ be positive integers such that $n>f+1>2$, and assume $Q$ is a quandle of cyclic type of order $n$ with $f$ fixed points. Then, the following hold.

1. Assume $n>2 f$.

(a) Then, $Q$ is connected.

(b) Moreover, there is only one such quandle, up to isomorphism. It occurs for $n=6$ and $f=2$. This quandle is $Q_{6}^{2}$ (see Table 1, above).

2. Assume $n \leqslant 2 f$.

(a) Then, $Q$ is not connected. 
(b) If $n=2 f$, there is only one such quandle, up to isomorphism. Its permutations are

$$
\begin{gathered}
\mu_{1}=\mu_{2}=\cdots=\mu_{f}=\left(\begin{array}{llll}
f+1 & f+2 & \cdots & 2 f
\end{array}\right) \\
\mu_{f+1}=\mu_{f+2}=\cdots=\mu_{2 f}=\left(\begin{array}{llll}
1 & 2 & \cdots & f
\end{array}\right) .
\end{gathered}
$$

Theorem 15. Let $n$ and $f$ be positive integers such that $n>f+1>2$ and $n \leqslant 2 f$ (corresponding to the non-connected case in Theorem 14). If $(n-f) \mid f$, there is a quandle of cyclic type of order $n$ with $f$ fixed points whose permutations are given as follows. For each $i$ such that $1 \leqslant i \leqslant \frac{n}{n-f}$,

$$
\begin{gathered}
\mu_{(i-1)(n-f)+1}=\mu_{(i-1)(n-f)+2}=\cdots=\mu_{i(n-f)}= \\
=\left(\begin{array}{llll}
i(n-f)+1 & i(n-f)+2 & \cdots & (i+1)(n-f)
\end{array}\right),
\end{gathered}
$$

with indices read $\bmod \frac{n}{n-f}$.

Theorem 16. Suppose $f$ is an integer strictly greater than 2 and $n$ a positive integer such that $f+2 \leqslant n \leqslant 2 f$. Consider a quandle of cyclic type of order $n$ and $f$ fixed points over the set $Q=\{1,2, \ldots, n\}$ with sequence of permutations $\mu_{i}$ with $i \in\{1,2, \ldots, n\}$. Assume further there is a $g_{0} \in Q$ such that $\mu_{i}\left(g_{0}\right)=g_{0}$, for any $i \in Q$. Then, the set $Q^{\prime}=Q \backslash\left\{g_{0}\right\}$ along with the sequence of permutations $\mu_{i}^{\prime}=\left.\mu_{i}\right|_{Q^{\prime}}$ for each $i \in Q^{\prime}$ defines a quandle of cyclic type of order $n-1$ with $f-1$ fixed points. We call this the extraction of the common fixed point $g_{0}$.

Theorem 17. Let $n$ be an integer greater than 2. Let $Q$ be the underlying set of a quandle whose permutations are denoted $\mu_{i}$, for each $i \in Q$. Let $g_{0} \notin Q$ and consider the set $Q^{\prime}=Q \cup\left\{g_{0}\right\}$. Suppose there is a permutation, $\mu$, of the elements of $Q$, such that $\mu \mu_{i}=\mu_{i} \mu$, for each $i \in Q$. Then, $Q^{\prime}$ along with the permutations

$$
\mu_{i}^{\prime}=\left(g_{0}\right) \mu_{i} \quad \text { for each } i \in Q \quad \text { and } \quad \mu_{g_{0}}^{\prime}=\left(g_{0}\right) \mu
$$

is a quandle with a common fixed point, $g_{0}$. We call this the adjoining of a common fixed point $g_{0}$.

Corollary 18. Let $(Q, \mu)$ be a quandle of cyclic type of order $n$ and $f$ fixed points with $(n-f) \mid f$ as in Theorem 15. Then any two permutations are either equal or move points from disjoint sets. So adjoining a common fixed point $g_{0}$ is accomplished by taking $\mu_{g_{0}}^{\prime}=\left(g_{0}\right) \mu_{i_{0}}$ by picking any $i_{0} \in Q$ and for any $j \in Q, \mu_{j}^{\prime}=\left(g_{0}\right) \mu_{j}$.

Furthermore, this procedure can be iterated indefinitely, giving rise to an infinite sequence of quandles $Q_{k}$ of cyclic type of order $n+k$ and $f+k$ fixed points such that $f+k+2 \leqslant n+k \leqslant 2(f+k)$. 


\subsection{Organization}

The Sections below are devoted to the proofs of these facts. In Section 2 we prove that quandles of cyclic type of order $n$ with $f$ fixed points in the range $n>2 f$ are connected (assertion 1.(a) in Theorem 14) and that for $n=2 f$ there is only one such quandle, up to isomorphism and that this quandle is not connected (assertion 2.(b) and assertion 2.(a) (for $n=2 f$ ) in Theorem 14). In Subsection 2.3 we prove that quandles of cyclic type of order $n$ and $f$ fixed points in the range $f+2 \leqslant n \leqslant 2 f$ are not connected; this is the 2.(a) part (for $n<2 f$ ) in Theorem 14. In Section 3 we prove that, up to isomorphism, there is only one quandle of cyclic type of order $n$ with $f$ fixed points in the range $n>2 f$. This quandle occurs for $n=6$ and $f=2$ and is known as the octahedron quandle (assertion 1.(b) in Theorem 14). This completes the proof of Theorem 14. In Section 4 we prove Theorems 15, 16, and 17. Finally, in Section 5 we collect a few questions for further research.

\section{Quandles of Cyclic Type with Several Fixed Points - First Properties and Examples}

In this Section, we state and prove a theorem about the structure of quandles of cyclic type with several fixed points. This theorem provides a number of conditions quandles of cyclic type of order $n$ with $f$ fixed points such that $n \geqslant 2 f$ must verify. This theorem is key to proceed to a classification of quandles of cyclic type.

\subsection{Associate Indices}

Quandles of cyclic type of order $n \geqslant 2 f$ have a very useful property, which is a consequence of the following proposition.

Proposition 19. Let $Q$ be a quandle of cyclic type of order $n$ with $f$ fixed points such that $n \geqslant 2 f$, and let $F_{k}=\left\{k, g_{k}^{1}, \ldots, g_{k}^{f-1}\right\}$ be the set of $f$ fixed points of $\mu_{k}$. Then $\mu_{g_{k}^{i}}(g)=g$, $\forall g \in F_{k}, \forall i \in\{1, \ldots, f-1\}$.

Proof. For each $i \in\{1, \ldots, f-1\}$ and for each $g \in F_{k} \backslash\left\{g_{k}^{i}\right\}$, we have

$$
\mu_{g_{k}^{i}}(g)=\mu_{\mu_{k}\left(g_{k}^{i}\right)}(g)=\mu_{k} \mu_{g_{k}^{i}} \mu_{k}^{-1}(g)=\mu_{k}\left(\mu_{g_{k}^{i}}(g)\right),
$$

that is, $\mu_{k}$ fixes $\mu_{g_{k}^{i}}(g)$ and hence $\mu_{g_{k}^{i}}(g) \in F_{k} \backslash\left\{g_{k}^{i}\right\}$, as $\mu_{g_{k}^{i}}\left(g_{k}^{i}\right)=g_{k}^{i}$. Therefore, the restriction of $\mu_{g_{k}^{i}}$ to $F_{k} \backslash\left\{g_{k}^{i}\right\}$ is a bijection from this set to itself. Thus, we must have $\mu_{g_{k}^{i}}(g)=g, \forall g \in F_{k} \backslash\left\{g_{k}^{i}\right\}$. Otherwise, $\mu_{g_{k}^{i}}$ would have a cycle of length $1<l \leqslant f-1=$ $\left|F_{k} \backslash\left\{g_{k}^{i}\right\}\right|$, that would also verify $l=n-f \geqslant 2 f-f=f$, which is a contradiction. Hence, the result follows.

We introduce the notions of associate indices and associate permutations.

Definition 20. Let $Q$ be a quandle of order $n$ with permutations denoted by $\mu_{k}, k \in$ $\{1, \ldots, n\}$. 
- If $i$ and $j$ are different indices such that $\mu_{i}(j)=j$ and $\mu_{j}(i)=i$, we say that $i$ and $j$ are associate indices;

- If $i$ and $j$ are associate indices then $\mu_{i}$ and $\mu_{j}$ are said associate permutations;

Corollary 21. With the terminology introduced in Definition 20, Proposition 19 states that, for each quandle of cyclic type whose order $n$ and number of fixed points $f$ satisfies $n \geqslant 2 f$, associate permutations have the same sets of fixed points.

In the sequel, we assume the order $n$ of any quandle of cyclic type to be greater than or equal to $2 f$, unless otherwise stated. Therefore, Proposition 19 always applies.

We now prove the main result of this section, which is a consequence of the previous results.

Corollary 22. Assume $n$ is the order of a quandle of cyclic type with $f$ fixed points. If $n \geqslant 2 f$, then $n$ is a multiple of $f$ i.e., $n=c f$ for some integer $c \geqslant 2$.

Proof. We show that if there is an index $s$ such that $\mu_{s}(k)=k$, for some $k$, then $s \in F_{k}$. For each $g \in F_{s} \backslash\{k\}$,

$$
\mu_{k}(g)=\mu_{\mu_{s}(k)}(g)=\mu_{s} \mu_{k} \mu_{s}^{-1}(g)=\mu_{s}\left(\mu_{k}(g)\right),
$$

that is, $\mu_{s}$ fixes $\mu_{k}(g)$ and hence $\mu_{k}(g) \in F_{s} \backslash\{k\}$, as $\mu_{k}(k)=k$. Therefore, the restriction of $\mu_{k}$ to $F_{s} \backslash\{k\}$ is a bijection from this set to itself. Arguing as in the proof of Proposition 19, we must have $\mu_{k}(g)=g, \forall g \in F_{s} \backslash\{k\}$, which implies that $F_{s}=F_{k}$, and in particular, $s \in F_{k}$. Thus, the sets of fixed points corresponding to two permutations are either equal or disjoint. Therefore, the order $n$ of a quandle of cyclic type with $f$ fixed points, with $n \geqslant 2 f$, has to be a multiple of $f$.

The following proposition is now an immediate consequence of our previous considerations.

Proposition 23. Given a quandle of cyclic type of order $n$ with $f$ fixed points and $n \geqslant$ $2 f>2$, " $i$ is associate to $j$ " generates an equivalence relation on the underlying set of the quandle.

Proof. The equivalence relation is " $i$ is associate to $j$ or $i=j$ ". Since $i$ is a fixed point of $\mu_{i}$, then $i \in F_{i}$. Moreover, any two sets $F_{i}$ and $F_{j}$ are either equal or disjoint, thus the result follows.

Example 24. $R_{4}$, the dihedral quandle of order 4, whose multiplication table is displayed in Table 2, is a quandle of cyclic type of order 4 with 2 fixed points.

\begin{tabular}{|l|llll|}
\hline$*$ & 1 & 2 & 3 & 4 \\
\hline 1 & 1 & 3 & 1 & 3 \\
2 & 4 & 2 & 4 & 2 \\
3 & 3 & 1 & 3 & 1 \\
4 & 2 & 4 & 2 & 4 \\
\hline
\end{tabular}

Table 2: $R_{4}$ multiplication table. 
By Proposition 23, "i is associate to j" generates an equivalence relation on $R_{4}$, which is also a congruence relation on this set, as it respects the binary operation of the quandle. In Table 3, we see the quotient of $R_{4}$ by this congruence relation, which we denote by $\sim$. This quotient is clearly isomorphic to $T_{2}$. In particular, $R_{4}$ is not simple since it admits a non-trivial quotient.

\begin{tabular}{|c|cc|}
\hline $\bar{*}$ & $\{1,3\}$ & $\{2,4\}$ \\
\hline$\{1,3\}$ & $\{1,3\}$ & $\{1,3\}$ \\
$\{2,4\}$ & $\{2,4\}$ & $\{2,4\}$ \\
\hline
\end{tabular}

Table 3: $R_{4} / \sim$ multiplication table.

\subsection{Connected Quandles of Cyclic Type of Order $n$ with $f$ Fixed Points in the Range $n \geqslant 2 f$ - First Properties and Example}

From this point on, we are only working with connected quandles of cyclic type of order $n \geqslant 2 f$. The two following propositions tell us whether a quandle of cyclic type of order $n \geqslant 2 f$ is connected or not.

Proposition 25. If $Q$ is a quandle of cyclic type of order $n$ with $f$ fixed points such that $n=2 f, Q$ is not connected.

Proof. Suppose $Q$ is a quandle of cyclic type of order $n$ with $f$ fixed points such that $n=2 f$ and let $i, j \in Q$ be two non-associate indices. Then $F_{i} \cup F_{j}=Q$. Moreover, since $n-f=2 f-f=f$, for any $a \in Q$, if $F_{i}$ (respect., $F_{j}$ ) is the set of fixed points of $\mu_{a}$, then $C_{a}=F_{j}$ (respect., $C_{a}=F_{i}$ ). Then, for each $a \in Q, \mu_{a}\left(F_{i}\right)=F_{i}$ and $\mu_{a}\left(F_{j}\right)=F_{j}$. Therefore, we conclude that $Q$ is not connected.

Proposition 26. Every quandle of cyclic type of order $n$ with $f$ fixed points such that $n>2 f$ is connected.

Proof. Suppose $Q$ is a quandle of cyclic type of order $n$ with $f$ fixed points such that $n=c f$, with $c \geqslant 3$ by Corollary 22. Let $i, j \in Q$, with $i \neq j$. If $i$ and $j$ are associate indices, then for any $k \notin F_{i}, i, j \in C_{k}$. Hence, there exists an integer $a \in\{1, \ldots, n-f-1\}$ such that $\mu_{k}^{a}(i)=j$. Now, assume $i$ and $j$ are not associate indices. Since there are at least three distinct sets of associate indices, there is at least one set $F_{k_{0}}$ such that both $i$ and $j$ do not belong to $F_{k_{0}}$. Therefore $i, j \in C_{k_{0}}$ and so there exists an integer $a \in\{1, \ldots, n-f-1\}$ such that $\mu_{k_{0}}^{a}(i)=j$. We conclude that $Q$ is connected.

Remark 27. In the sequel, we assume the order $n$ of any quandle to be greater than $2 f$, unless otherwise stated. In this condition, all our quandles of cyclic type are connected.

We now use some of the equalities $\mu_{\mu_{i}(j)}=\mu_{i} \mu_{j} \mu_{i}^{-1}$ the permutations defining these quandles have to verify to derive a number of conditions these quandles have to satisfy in order to be cyclic. 
Theorem 28. Consider a quandle of cyclic type of order $n$ with $f$ fixed points such that $n>2 f$. Modulo isomorphism, its sequence of permutations satisfies the following conditions.

1. $\mu_{n}=\left(\begin{array}{lllll}1 & 2 & 3 & \cdots & n-f\end{array}\right)(n-f+1) \cdots(n-1)(n)$;

2. if $h$ and $h^{\prime}$ are associate indices then $\mu_{h}=\mu_{h^{\prime}}^{l_{h, h^{\prime}}}$, where $G C D\left(n-f, l_{h, h^{\prime}}\right)=1$, $1 \leqslant l_{h, h^{\prime}}<n-f$

3. $\mu_{k}=\mu_{n}^{k} \mu_{n-f} \mu_{n}^{-k}$, for all $1 \leqslant k \leqslant n-f$;

4. $\mu_{n-f} \mu_{a} \mu_{n-f}^{-1}=\mu_{n-f}^{\mu_{n-f}(a)} \mu_{n-f} \mu_{n}^{-\mu_{n-f}(a)}, \forall a \in F_{n}$;

5. $\mu_{n-f}^{-1} \mu_{a} \mu_{n-f}=\mu_{n-f}^{\mu_{n-f}^{-1}(a)} \mu_{n-f} \mu_{n}^{-\mu_{n-f}^{-1}(a)}, \forall a \in F_{n}$;

6. $\forall m \in\{1, \ldots, n-f\} \backslash\left\{\mu_{n-f}^{-1}(n-f+1), \ldots, \mu_{n-f}^{-1}(n)\right\}$, there exists an integer $1 \leqslant$ $k_{m}<n-f$ such that $\mu_{n}^{-\mu_{n-f}(m)} \mu_{n-f} \mu_{n}^{m}=\sigma \tau^{k_{m}}$, where $\sigma$ is a permutation of $F_{n-f}$ and $\tau$ is the cycle of length $n-f$ in $\mu_{n-f}$.

Proof.

1. We can assume that $\mu_{n}$ is given by $(123 \cdots n-f)(n-f+1) \cdots(n-1)(n)$ without loss of generality. If necessary, we may relabel the indices. This expression for $\boldsymbol{\mu}_{\boldsymbol{n}}$ will be assumed in the sequel - except for Subsection 2.3.

2. Suppose $h$ and $h^{\prime}$ are associate indices and let $F_{h}=\left\{h, h^{\prime}, g_{h}^{1}, \ldots, g_{h}^{f-2}\right\}$. Therefore, we have that

$$
\mu_{h}=\left(h_{1} \ldots h_{n-f}\right)(h)\left(h^{\prime}\right)\left(g_{h}^{1}\right) \cdots\left(g_{h}^{f-2}\right)
$$

and

$$
\mu_{h^{\prime}}=\left(h_{1}^{\prime} \ldots h_{n-f}^{\prime}\right)(h)\left(h^{\prime}\right)\left(g_{h}^{1}\right) \cdots\left(g_{h}^{f-2}\right),
$$

and hence

$$
\begin{gathered}
\mu_{h^{\prime}}=\mu_{\mu_{h}\left(h^{\prime}\right)}=\mu_{h} \mu_{h^{\prime}} \mu_{h}^{-1} \Leftrightarrow \mu_{h^{\prime}} \mu_{h}=\mu_{h} \mu_{h^{\prime}} \Leftrightarrow \\
\Leftrightarrow\left(h_{1} \ldots h_{n-f}\right)\left(h_{1}^{\prime} \ldots h_{n-f}^{\prime}\right)=\left(h_{1}^{\prime} \ldots h_{n-f}^{\prime}\right)\left(h_{1} \ldots h_{n-f}\right),
\end{gathered}
$$

that is, the two cycles $\left(h_{1} \ldots h_{n-f}\right)$ and $\left(h_{1}^{\prime} \ldots h_{n-f}^{\prime}\right)$ commute in

$$
S_{\left\{h_{1}, \ldots, h_{n-f}\right\}}=S_{\left\{h_{1}^{\prime}, \ldots, h_{n-f}^{\prime}\right\}} \text {. }
$$

Thus, $\mu_{h}=\mu_{h^{\prime}}^{l_{h, h^{\prime}}}$ (see [16], for instance), where $l_{h, h^{\prime}}$ satisfies $G C D\left(n-f, l_{h, h^{\prime}}\right)=1$, $1 \leqslant l_{h, h^{\prime}}<n-f$ (otherwise $\mu_{h}$ would not have a cycle of length $n-f$ ).

3. First, we note that $\mu_{1}=\mu_{\mu_{n}(n-f)}=\mu_{n} \mu_{n-f} \mu_{n}^{-1}$. If $\mu_{k}=\mu_{n}^{k} \mu_{n-f} \mu_{n}^{-k}$ then

$$
\mu_{k+1}=\mu_{\mu_{n}(k)}=\mu_{n} \mu_{k} \mu_{n}^{-1}=\mu_{n} \mu_{n}^{k} \mu_{n-f} \mu_{n}^{-k} \mu_{n}^{-1}=\mu_{n}^{k+1} \mu_{n-f} \mu_{n}^{-(k+1)},
$$


(where we read the free indices modulo $n-f$ ), whence we proved 3. by induction.

4. Let $a \in F_{n}$ and assume $\mu_{n-f}(a) \notin F_{n}$. Let $i \notin F_{n}$. On one hand, we have, by assertions 2. and 3.,

$$
\mu_{\mu_{i}(a)}=\mu_{i} \mu_{a} \mu_{i}^{-1}=\mu_{n}^{i} \mu_{n-f} \mu_{n}^{-i} \mu_{a} \mu_{n}^{i} \mu_{n-f}^{-1} \mu_{n}^{-i}=\mu_{n}^{i} \mu_{n-f} \mu_{a} \mu_{n-f}^{-1} \mu_{n}^{-i} .
$$

On the other hand, again by assertion 3.,

$$
\mu_{i}(a)=\mu_{n}^{i} \mu_{n-f} \mu_{n}^{-i}(a)=\mu_{n}^{i} \mu_{n-f}(a)=\mu_{n}^{i} \mu_{n}^{\mu_{n-f}(a)}(n-f)=\mu_{n}^{i+\mu_{n-f}(a)}(n-f),
$$

which implies that

$$
\mu_{\mu_{i}(a)}=\mu_{\mu_{n}^{i+\mu_{n-f}(a)}(n-f)}=\mu_{n}^{i} \mu_{n}^{\mu_{n-f}(a)} \mu_{n-f} \mu_{n}^{-\mu_{n-f}(a)} \mu_{n}^{-i}
$$

Combining 1 and 2, we get

$$
\mu_{n-f} \mu_{a} \mu_{n-f}^{-1}=\mu_{n-f}^{\mu_{n-f}(a)} \mu_{n-f} \mu_{n}^{-\mu_{n-f}(a)} .
$$

We now prove the following lemma, which completes the proof of assertion 4 ..

Lemma 29. Given $a \in F_{n}, \mu_{n-f}(a) \notin F_{n}$.

Proof. Suppose $\mu_{n-f}(a) \in F_{n}$. Then, for $1 \leqslant k \leqslant n-f$,

$$
\mu_{k}(a)=\mu_{n}^{k} \mu_{n-f} \mu_{n}^{-k}(a)=\mu_{n}^{k} \mu_{n-f}(a)=\mu_{n-f}(a) .
$$

This would force the pairs of associate permutations from $\mu_{1}$ to $\mu_{n-f}$ to be equal to each other. In fact, by assertion 2., if two associate permutations have the same image at a point belonging to their non-singular cycles, these permutations have to be the same. Suppose, now, that for a certain index $b \in F_{n}, \mu_{n-f}(b) \notin F_{n}$. Then, for $1 \leqslant k \leqslant n-f$,

$$
\mu_{k}(b)=\mu_{n}^{k} \mu_{n-f} \mu_{n}^{-k}(b)=\mu_{n}^{k} \mu_{n-f}(b)=\mu_{n-f}(b)+k,
$$

which would force the pairs of permutations whose indices are associate from $\mu_{1}$ to $\mu_{n-f}$ to be all different from each other, which is a contradiction. Therefore, $\mu_{n-f}\left(F_{n}\right)=F_{n}$. But $\mu_{n-f}$ does not fix any element from $F_{n}$ since $F_{n} \cap F_{n-f}=\emptyset$. Then, this implies that $\mu_{n-f}$ has a cycle of length at most $f$, which is again a contradiction, since $n-f>f$. Hence, $\mu_{n-f}(a) \notin F_{n}$.

5. Let $a \in F_{n}$ and let $i \notin F_{n}$ be the index such that $\mu_{n-f}(i)=a$. Note that otherwise $i$ would take up the role of $a$ in Lemma 29 and $a$ would not belong to $F_{n}$. Then,

$$
\mu_{a}=\mu_{\mu_{n-f}(i)}=\mu_{n-f} \mu_{i} \mu_{n-f}^{-1}=\mu_{n-f} \mu_{n}^{i} \mu_{n-f} \mu_{n}^{-i} \mu_{n-f}^{-1} .
$$

Since $i=\mu_{n-f}^{-1}(a)$, we conclude that $\mu_{n-f}^{-1} \mu_{a} \mu_{n-f}=\mu_{n-f}^{\mu_{n-1}^{-1}(a)} \mu_{n-f} \mu_{n}^{-\mu_{n-f}^{-1}(a)}$. 
6. Let $m$ be any index belonging to the set $\{1, \ldots, n-f\}$ such that the index $\mu_{n-f}(m)$ belongs to the set $\{1, \ldots, n-f\} \backslash\left\{\mu_{n-f}(n), \ldots, \mu_{n-f}(n-f+1)\right\}$. Then, by assertion 3.,

$$
\mu_{\mu_{n-f}(m)}=\mu_{n-f} \mu_{m} \mu_{n-f}^{-1}=\mu_{n-f} \mu_{n}^{m} \mu_{n-f} \mu_{n}^{-m} \mu_{n-f}^{-1} .
$$

Since we also have $\mu_{\mu_{n-f}(m)}=\mu_{n-f}^{\mu_{n-f)}} \mu_{n-f} \mu_{n}^{-\mu_{n-f}(m)}$, then

$$
\mu_{n-f} \mu_{n}^{m} \mu_{n-f} \mu_{n}^{-m} \mu_{n-f}^{-1}=\mu_{n-f}^{\mu_{n-f}(m)} \mu_{n-f} \mu_{n}^{-\mu_{n-f}(m)},
$$

which is equivalent to having

$$
\left(\mu_{n}^{-\mu_{n-f}(m)} \mu_{n-f} \mu_{n}^{m}\right) \mu_{n-f}=\mu_{n-f}\left(\mu_{n}^{-\mu_{n-f}(m)} \mu_{n-f} \mu_{n}^{m}\right),
$$

that is, $\mu_{n}^{-\mu_{n-f}(m)} \mu_{n-f} \mu_{n}^{m}$ and $\mu_{n-f}$ commute in $S_{n}$. The number of elements in the centralizer of $\mu_{n-f}$ in $S_{n},\left|C_{S_{n}}\left(\mu_{n-f}\right)\right|$, is given by

$$
\left|C_{S_{n}}\left(\mu_{n-f}\right)\right|=\frac{\left|S_{n}\right|}{\left|\mu_{n-f}^{S_{n}}\right|}, \quad \text { see [16], for instance, }
$$

where $\left|\mu_{n-f}^{S_{n}}\right|$ denotes the number of elements of $S_{n}$ with the same pattern as $\mu_{n-f}$. In fact, we have

$$
\left|\mu_{n-f}^{S_{n}}\right|=\frac{n(n-1) \ldots(f+1)}{n-f}=\frac{n !}{(n-f) f !},
$$

and since $\left|S_{n}\right|=n$ !, we conclude that $\left|C_{S_{n}}\left(\mu_{n-f}\right)\right|=(n-f) f$ !. However, we know exactly what these $(n-f) f$ ! permutations are. Let $\tau$ be the cycle of length $n-f$ of $\mu_{n-f}$. Indeed, $\tau^{k}$ commutes with $\mu_{n-f}, \forall k \in\{1, \ldots, n-f\}$. Moreover, any permutation of the $f$ fixed points of $\mu_{n-f}$ commutes with $\mu_{n-f}$. The former type of permutation $\tau^{k}$ only moves elements within $C_{n-f}$ whereas the latter type of permutation only moves elements within $F_{n-f}$. Composing permutations from these two commuting types of permutations, we get a total of $(n-f) f$ ! permutations commuting with $\mu_{n-f}$, which is precisely the number of permutations we found before. Therefore, we may conclude that $\forall m \in\{1, \ldots, n-f\} \backslash\left\{\mu_{n-f}^{-1}(n-f+1), \ldots, \mu_{n-f}^{-1}(n)\right\}$, there exists an integer $1 \leqslant k_{m}<n-f$ such that $\mu_{n}^{-\mu_{n-f}(m)} \mu_{n-f} \mu_{n}^{m}=\sigma \tau^{k_{m}}$, where $\sigma$ is a permutation of $F_{n-f}$ and $\tau$ is the cycle of length $n-f$ in $\mu_{n-f}$.

Corollary 30. Assertions 1., 2., and 3. in Theorem 28 are still valid if $n=2 f$.

Proof. Omitted.

This allows us to classify quandles of cyclic type of order $2 f$ with $f$ fixed points

Corollary 31. For any integer $f>1$, there is only one quandle of cyclic type of order $2 f$ with $f$ fixed points, up to isomorphism. Moreover, such quandle is not connected. 
Proof. Let $f$ be as in the statement. We first note that, should it exist, the indicated quandle is not connected via Proposition 25. We will next prove (1.) that there is such a quandle; and then (2.) that any such quandle is isomorphic to the one in 1.

1. Consider the sequence of permutations

$$
\begin{aligned}
& \mu_{i}=(1)(2) \cdots(f)(f+1 \quad f+2 \quad f+3 \quad \cdots \quad 2 f) \quad \text { for } i=1,2, \ldots, f \text {; } \\
& \mu_{j}=(f+1)(f+2) \cdots(2 f)(1 \quad 2 \quad 3 \quad \cdots \quad f) \quad \text { for } j=f+1, f+2, \ldots, 2 f .
\end{aligned}
$$

Note that $\mu_{i}$ 's and $\mu_{j}$ 's commute among themselves and with one another, since they are either equal or move points from disjoint sets. Then, for any $i, i^{\prime} \in\{1,2, \ldots, f\}$ and $j, j^{\prime} \in\{f+1, f+2, \ldots, 2 f\}$, we have

$$
\begin{gathered}
\mu_{i}\left(i^{\prime}\right)=i^{\prime} \Longrightarrow \mu_{i^{\prime}}=\mu_{i} \mu_{i^{\prime}} \mu_{i}^{-1}=\mu_{i} \\
\mu_{i}(j)=j+1(\text { with } 2 f+1=f+1) \Longrightarrow \mu_{j+1}=\mu_{i} \mu_{j} \mu_{i}^{-1}=\mu_{j} \\
\mu_{j}\left(j^{\prime}\right)=j^{\prime} \Longrightarrow \mu_{j^{\prime}}=\mu_{j} \mu_{j^{\prime}} \mu_{j}^{-1}=\mu_{j} \\
\mu_{j}(i)=i+1(\text { with } f+1=f) \Longrightarrow \mu_{i+1}=\mu_{j} \mu_{i} \mu_{j}^{-1}=\mu_{i} .
\end{gathered}
$$

Therefore, the indicated sequence of permutations defines a quandle. Moreover, this is a quandle of cyclic type of order $2 f$ with $f$ fixed points.

2. Now consider a quandle of cyclic type of order $2 f$ and $f$ fixed points, along with its permutations, $\mu_{i}^{\prime}$ for $i=1,2, \ldots, 2 f$. According to 1 . in Theorem 28 and Corollary 30

$$
\mu_{2 f}^{\prime}=(f+1)(f+2) \cdots(2 f)(1 \quad 2 \quad 3 \quad \cdots \quad f),
$$

whose set of fixed points is

$$
F_{2 f}=\{f+1, f+2, \ldots, 2 f\}=F_{2 f-1}=\cdots=F_{f+1} .
$$

There are two distinct sets of fixed points (see proof of Proposition 25), so the other one is

$$
F_{1}=\{1,2, \ldots, f\}=F_{2}=\cdots=F_{f} .
$$

Then

$$
\mu_{1}^{\prime}=(1)(2) \cdots(f)\left(g_{1} \quad g_{2} \quad g_{3} \quad \cdots \quad g_{f}\right),
$$

where $\left(\begin{array}{lllll}g_{1} & g_{2} & g_{3} & \cdots & g_{f}\end{array}\right)$ is a cyclic permutation of $\{f+1, f+2, \ldots, 2 f\}$. For any $i \in\{1,2, \ldots, f\}$, we have

$$
\mu_{2 f}^{\prime}(i)=i+1(\text { with } f+1=1) \Longrightarrow \mu_{i+1}^{\prime}=\mu_{2 f}^{\prime} \mu_{i}^{\prime} \mu_{2 f}^{\prime}{ }^{-1}=\mu_{i}^{\prime} .
$$

Therefore,

$$
\mu_{i}^{\prime}=\mu_{1}^{\prime}=(1)(2) \cdots(f)\left(g_{1} \quad g_{2} \quad g_{3} \quad \cdots \quad g_{f}\right) \quad \text { for any } i \in\{1,2, \ldots, f\} .
$$


Also, for any $i \in\{1,2, \ldots, f\}$, we have

$$
\mu_{1}^{\prime}(f+i)=f+i+1(\text { with } 2 f+1=f+1) \Longrightarrow \mu_{f+i+1}^{\prime}=\mu_{1}^{\prime} \mu_{f+i}^{\prime} \mu_{1}^{\prime-1}=\mu_{f+i}^{\prime} .
$$

Therefore,

$\mu_{f+i}^{\prime}=\mu_{2 f}^{\prime}=(f+1)(f+2) \cdots(2 f)(1 \quad 2 \quad 3 \quad \cdots \quad f) \quad$ for any $i \in\{1,2, \ldots, f\}$.

Finally, consider the permutation, $\alpha$, of $\{1,2, \ldots, f, f+1, \ldots, 2 f\}$ given by

$$
\alpha=(1)(2) \cdots(f)\left(g_{1} f+1\right)\left(g_{2} f+2\right) \cdots\left(g_{f} 2 f\right),
$$

where, in case $g_{i}=f+i,\left(g_{i} f+i\right)$ is to be read $\left(g_{i}\right)$, a fixed point. Then, for any $i \in\{1,2, \ldots, f, f+1, \ldots, 2 f\}$,

$$
\mu_{\alpha(i)}^{\prime}=\alpha \mu_{i} \alpha^{-1}
$$

Thus, $\alpha$ is a quandle isomorphism between the quandle here and the quandle in 1.

The proof of Corollary 31 establishes Assertion 2.(b) in Theorem 14.

\subsection{Quandles of Cyclic Type of Order $n$ with $f$ Fixed Points in the Range $f+2 \leqslant n \leqslant 2 f$ are not Connected}

Theorem 32. Cyclic quandles of order $n$ with $f$ fixed points such that $f+2 \leqslant n \leqslant 2 f$ are not connected.

Proof. By Proposition 25, we know this is true for $n=2 f$. Now, let $Q$ be a cyclic quandle of order $n$ with $f$ fixed points such that $f+2 \leqslant n \leqslant 2 f-1$. We assume, without loss of generality, that $\mu_{n}=\left(\begin{array}{lll}1 & 2 \ldots n-f\end{array}\right)(n-f+1)(n-f+2) \ldots(n)$, see Assertion 1. in Theorem 28. Given $j, k \in F_{n}=\{n-f+1, n-f+2, \ldots, n\}$,

$$
\mu_{k}(j)=\mu_{\mu_{n}(k)}(j)=\mu_{n} \mu_{k} \mu_{n}^{-1}(j)=\mu_{n}\left(\mu_{k}(j)\right),
$$

that is, $\mu_{n}$ fixes $\mu_{k}(j)$. Therefore, we have that $\mu_{k}(j) \in F_{n}$ for any $j, k \in F_{n}$. Thus, $F_{n}$ is a subquandle of $Q$. Now, if this subquandle has constant profile, then the common pattern is that of $\left.\mu_{n}\right|_{F_{n}}=(n-f+1)(n-f+2) \ldots(n)$, hence $F_{n}$ as a quandle is the trivial quandle on $f$ elements. In particular, it is not connected. If $F_{n}$ as a quandle has not constant profile, then, by Proposition 10, it is not connected. In either case, this subquandle is not connected and hence there is a finite family of minimal disjoint sets $F_{n}^{i}, i \in\{1,2, \ldots, d\}$, such that $\bigcup_{i} F_{n}^{i}=F_{n}$ and $\mu_{g}\left(F_{n}^{i}\right)=F_{n}^{i}, \forall i, \forall g \in F_{n}$, which correspond to the (minimal) connected components of $F_{n}$, as a quandle. We also note that $C_{n} \cap F_{n}=\emptyset, C_{n} \cup F_{n}=Q$ and $\mu_{g}\left(C_{n}\right)=C_{n}, \forall g \in F_{n}$. Now, since $\left|C_{n}\right|=n-f<f=\left|F_{1}\right|, \mu_{1}$ must fix some point $a_{0} \in F_{n}$, i.e., $a_{0} \in F_{n} \cap F_{1}$.

Lemma 33. Let $a \in F_{n}$. Assume there exist $i_{0} \in\{1,2, \ldots, n-f\}$ such that: 
1. $a \in F_{i_{0}}$. Then $a \in F_{i}$ for each $i \in\{1,2, \ldots, n-f\}$.

2. $a \notin F_{i_{0}}$. Then $a \notin F_{i}$ for each $i \in\{1,2, \ldots, n-f\}$.

Proof. $\quad$ 1. Pick $i \in\{1,2, \ldots, n-f\}$ Then $i=\mu_{n}^{n-f+i-i_{0}}\left(i_{0}\right)$. Then

$$
\begin{aligned}
\mu_{i}(a) & =\mu_{\mu_{n}^{n-f+i-i_{0}}\left(i_{0}\right)}(a)=\mu_{n}^{n-f+i-i_{0}} \mu_{i_{0}} \mu_{n}^{-\left(n-f+i-i_{0}\right)}(a)=\mu_{n}^{n-f+i-i_{0}} \mu_{i_{0}}(a)= \\
& =\mu_{n}^{n-f+i-i_{0}}(a)=a .
\end{aligned}
$$

2. Assume to the contrary and suppose there is $i_{1} \in\{1,2, \ldots, n-f\}$ such that $\mu_{i_{1}}(a)=$ a. Then, by $1 ., \mu_{i}(a)=a$, for any $i \in\{1,2, \ldots, n-f\}$ which conflicts with $\mu_{i_{0}}(a) \neq a$.

Thus, the $a_{0} \in F_{1} \cap F_{n}$ above, satisfies, thanks to Lemma 33, $a_{0} \in F_{n} \cap F_{1} \cap F_{2} \cap \cdots \cap$ $F_{n-f}$.

Set

$$
A=\left\{\mu_{s}\left(a_{0}\right) \mid s \in\{1,2, \ldots, n\}\right\}=\left\{\mu_{j}\left(a_{0}\right) \mid j \in\{n-f+1, n-f+2, \ldots, n\}\right\} \subset F_{n}^{1},
$$

since $a_{0} \in F_{n} \cap F_{1} \cap F_{2} \cap \cdots \cap F_{n-f}$. In particular, $1 \leqslant|A| \leqslant f$.

For each $i \in\{1,2, \ldots, n-f\}$, here is the behaviour of $A$ under $\mu_{i}$. Let $j \in\{n-f+$ $1, n-f+2, \ldots, n\}$

$$
\mu_{i}\left(\mu_{j}\left(a_{0}\right)\right)=\mu_{i}\left(\mu_{j} \mu_{i}^{-1}\left(\mu_{i}\left(\left(a_{0}\right)\right)\right)\right)=\mu_{i}\left(\mu_{j} \mu_{i}^{-1}\left(\left(a_{0}\right)\right)\right)=\mu_{\mu_{i}(j)}\left(a_{0}\right) \in A .
$$

Furthermore, for $j, j^{\prime} \in\{n-f+1, n-f+2, \ldots, n\}$ such that $\mu_{j}\left(a_{0}\right) \neq \mu_{j^{\prime}}\left(a_{0}\right)$, then $\mu_{i}\left(\mu_{j}\left(a_{0}\right)\right) \neq \mu_{i}\left(\mu_{j^{\prime}}\left(a_{0}\right)\right)$, for any $i \in\{1,2, \ldots, n-f\}$. Then, for any $i \in\{1,2, \ldots, n-f\}$, $\mu_{i}$ restricted to $A$ is a bijection.

1. If $A=\left\{a_{0}\right\}$, then $\mu_{s}\left(a_{0}\right)=a_{0}$, for any $s \in\{1,2, \ldots, n\}$, so $A$ is a connected component of $Q$. Since $Q$ has more than one element then $Q$ is not connected.

2. Assume $|A|>1$.

(a) Assume further that, for each $i \in\{1,2, \ldots, n-f\}, \mu_{i}$ moves at least one element from $A$, say $\mu_{j_{0}}\left(a_{0}\right)$, for some $j_{0} \in\{n-f+1, n-f+2, \ldots, n\}$ - recall Lemma 33. Then,

$$
\mu_{i}\left(\mu_{j_{0}}\left(a_{0}\right)\right) \in A \backslash\left\{\mu_{j_{0}}\left(a_{0}\right)\right\} .
$$

Since $\mu_{i}$ has a cycle of length $n-f, C_{i}$, then $C_{i} \subset A$, for any $i \in\{1,2, \ldots, n-f\}$. In particular, in this case, if $b \notin A$, then $\mu_{i}(b)=b$, for any $i \in\{1,2, \ldots, n-f\}$. Since $A \subset F_{n}^{1}$, then $F_{n}^{1}$ is a connected component of $Q$. Since $C_{n} \cap F_{n}^{1}=\emptyset$ and $C_{n} \subset Q$, then $Q$ is not connected.

(b) Assume now that for each $i \in\{1,2, \ldots, n-f\}, \mu_{i}$ fixes any element of $A$ again, recall Lemma 33. That is, for each $j \in\{n-f+1, n-f+2, \ldots, n\}$,

$$
\mu_{i}\left(\mu_{j}\left(a_{0}\right)\right)=\mu_{j}\left(a_{0}\right) .
$$


i. If $A=F_{n}^{1}$, then we are done, arguing that $F_{n}^{1}$ is a connected component inside $Q$ which has fewer elements than $Q$.

ii. If $A \subsetneq F_{n}^{1}$, then since $F_{n}^{1}$ is a minimal component of $F_{n}$, there exist $j_{0}, j_{1} \in$ $\{n-f+1, n-f+2, \ldots n\}$ such that

$$
\mu_{j_{1}}\left(\mu_{j_{0}}\left(a_{0}\right)\right) \in F_{n}^{1} \backslash A \text {. }
$$

Let $B_{1}=\left\{\mu_{j_{1}}\left(\mu_{j_{0}}\left(a_{0}\right)\right) \in F_{n}^{1} \backslash A \mid j_{0}, j_{1} \in\{n-f+1, n-f+2, \ldots n\}\right\}$. Note that if for some $i \in\{1,2, \ldots, n-f\}$, and for some $j_{0}, j_{1} \in\{n-f+1, n-$ $f+2, \ldots n\}$ such that $\mu_{j_{1}}\left(\mu_{j_{0}}\left(a_{0}\right)\right) \in F_{n}^{1} \backslash A$, we had $\mu_{i}\left(\mu_{j_{1}}\left(\mu_{j_{0}}\left(a_{0}\right)\right)\right) \in A$, then $\mu_{j_{1}}\left(\mu_{j_{0}}\left(a_{0}\right)\right) \in A$, since $\mu_{i}$ restricted to $A$ is a bijection. Therefore, $\mu_{i}$ restricted to $B_{1}$ is a bijection.

Set

$$
A_{1}:=A \cup B_{1} .
$$

Go back to 2. ("Assume $|A|>1$...") with $A_{1}$ taking up the role of $A$. Iterate the procedure. Since $Q$ is a finite quandle, this procedure has to finish after a finite number of steps, say $k$, with $A\left(=A_{k}\right)=F_{n}^{1}$.

The proof is complete.

\section{Classification of Quandles of Cyclic Type of order $n$ with $f$ Fixed Points in the Range $n>2 f$}

In this Section, we classify quandles of cyclic type of order $n$ with $f$ fixed points such that $n>2 f$. Specifically, we prove that there is only one such quandle such that $n>2 f$, up to isomorphism. In this range, this quandle occurs only for $n=6$ and $f=2$. This quandle is $Q_{6}^{2}$, up to isomorphism. The proof of this fact establishes Assertion 1.(b) in Theorem 14. This is the main goal of the current Section.

In Subsection 3.1, we prove a number of propositions and lemmas that we use in subsequent subsections. In Subsection 3.2, we show that there are no quandles of cyclic type of order $n$ with $f$ fixed points such that $n=3 f$ for $f>2$ and we prove that the only quandle of cyclic type of order 6 with 2 fixed points, up to isomorphism, is $Q_{6}^{2}$. In Subsection 3.3, we show that there are no quandles of cyclic type of order $n$ with $f$ fixed points such that $n=c f$ for $c>3$. Finally, in Subsection 3.4, we collect the results from the preceding subsections to prove Assertion 1.(b) in Theorem 14. We also show that $Q_{6}^{2}$ is not simple.

In this Section, the results apply only to quandles of cyclic type of order $n$ with $f$ fixed points such that $n>2 f$.

\subsection{Auxiliary Results}

In this Subsection, we state and prove a number of results about the structure of quandles of cyclic type of order $n$ with $f$ fixed points such that $n>2 f$. These results are used in the following Subsections. 
Proposition 34. The associate indices to $n-f$ are $i\left(\frac{n-f}{f}\right), i \in\{1, \ldots, f-1\}$.

Proof. We first prove that, if $a, b \in F_{n-f}$, then $b-a \in F_{n-f} \bmod n-f$. Since $a, b \in F_{n-f}$, $\mu_{a}$ fixes $b$ (Proposition 19) and hence, reading $b-a \bmod n-f$ and using Assertion 3. in Theorem 28,

$$
b=\mu_{a}(b)=\mu_{n}^{a} \mu_{n-f} \mu_{n}^{-a}(b)=\mu_{n}^{a} \mu_{n-f}(b-a)=\mu_{n-f}(b-a)+a \Leftrightarrow b-a=\mu_{n-f}(b-a),
$$

that is, $\mu_{n-f}$ fixes $b-a$, implying that $b-a \in F_{n-f}$ (where $b-a$ is read modulo $n-f$ ). Now, let the indices in $F_{n-f}=\left\{g_{n-f}^{1}, \ldots, g_{n-f}^{f}\right\}$ be labelled in such a way that $g_{n-f}^{i}<g_{n-f}^{i+1}$, $\forall i \in\{1, \ldots, f-1\}$. In particular, we have $g_{n-f}^{f}=n-f$. Suppose these indices are not equally spaced modulo $n-f$. Therefore, there is an index $1 \leqslant j_{0} \leqslant f$ such that $g_{n-f}^{j_{0}}-g_{n-f}^{j_{0}-1} \geqslant g_{n-f}^{i}-g_{n-f}^{i-1}, \forall i \in\{1, \ldots, f\}$, and there is another index $1 \leqslant k_{0} \leqslant f$ such that $g_{n-f}^{k_{0}}-g_{n-f}^{k_{0}-1} \leqslant g_{n-f}^{i}-g_{n-f}^{i-1}, \forall i \in\{1, \ldots, f\}$, where we take $g_{n-f}^{0}:=0$. Moreover, $g_{n-f}^{j_{0}}-g_{n-f}^{j_{0}-1}>g_{n-f}^{k_{0}}-g_{n-f}^{k_{0}-1}$. Now, by the result we have just proved, $g_{n-f}^{k_{0}}-g_{n-f}^{k_{0}-1}$ belongs to $F_{n-f}$, as well as $g_{n-f}^{j_{0}}-\left(g_{n-f}^{k_{0}}-g_{n-f}^{k_{0}-1}\right)$. However, we have $g_{n-f}^{j_{0}-1}<g_{n-f}^{j_{0}}-\left(g_{n-f}^{k_{0}}-g_{n-f}^{k_{0}-1}\right)<$ $g_{n-f}^{j_{0}}$, which is a contradiction, since $g_{n-f}^{j_{0}-1}$ and $g_{n-f}^{j_{0}}$ are consecutive indices in $F_{n-f}$. Hence, the indices in $F_{n-f}$ are equally spaced modulo $n-f$, and the associate indices to $n-f$ are $i\left(\frac{n-f}{f}\right), i \in\{1, \ldots, f-1\}$.

Corollary 35. For each index $i \in\{1, \ldots, n-f\}, F_{i}=\left\{i+j\left(\frac{n-f}{f}\right): 1 \leqslant j \leqslant f\right\}=$ $i+F_{n-f}$, where each index $i+j\left(\frac{n-f}{f}\right)$ is read modulo $n-f$.

Proof. Given $i \in\{1, \ldots, n-f\}$ and $j \in\{1, \ldots, f\}$, we prove $i+j\left(\frac{n-f}{f}\right)$ is a fixed point of $\mu_{i}$. In fact,

$$
\begin{aligned}
\mu_{i}\left(i+j\left(\frac{n-f}{f}\right)\right) & =\mu_{n}^{i} \mu_{n-f} \mu_{n}^{-i}\left(i+j\left(\frac{n-f}{f}\right)\right)=\mu_{n}^{i} \mu_{n-f}\left(j\left(\frac{n-f}{f}\right)\right)=\mu_{n}^{i}\left(j\left(\frac{n-f}{f}\right)\right)= \\
& =i+j\left(\frac{n-f}{f}\right) .
\end{aligned}
$$

Clearly, for $1 \leqslant j<j^{\prime} \leqslant f$, we have $i+j\left(\frac{n-f}{f}\right) \neq i+j^{\prime}\left(\frac{n-f}{f}\right) \bmod n-f$. Hence, we conclude that $F_{i}=\left\{i+j\left(\frac{n-f}{f}\right): 1 \leqslant j \leqslant f\right\}$, where each $i+j\left(\frac{n-f}{f}\right)$ is read modulo $n-f$.

Corollary 35 along with Proposition 19 and Corollary 22 tell us exactly what are the associate indices in a quandle of cyclic type with $f$ fixed points of order $n>2 f$.

Lemma 36. Given distinct indices $a, b \in F_{n}, \mu_{n-f}(b)-\mu_{n-f}(a) \in F_{n-f}$, where this index is read modulo $n-f$. 
Proof. Let $a, b \in F_{n}$. By assertion 2. in Theorem 28, we have $\mu_{b}=\mu_{a}^{l_{b, a}}$. Hence, using assertion 4. in Theorem 28,

$$
\begin{gathered}
\mu_{n-f}^{\mu_{n-f}(a)} \mu_{n-f}^{l_{b, a}} \mu_{n}^{-\mu_{n-f}(a)}=\left(\mu_{n}^{\mu_{n-f}(a)} \mu_{n-f} \mu_{n}^{-\mu_{n-f}(a)}\right)^{l_{b, a}}=\left(\mu_{n-f} \mu_{a} \mu_{n-f}^{-1}\right)^{l_{b, a}}= \\
=\mu_{n-f} \mu_{a}^{l_{b, a}} \mu_{n-f}^{-1}=\mu_{n-f} \mu_{b} \mu_{n-f}^{-1}=\mu_{n}^{\mu_{n-f}(b)} \mu_{n-f} \mu_{n}^{-\mu_{n-f}(b)},
\end{gathered}
$$

which implies, by assertion 3. in Theorem 28, that

$$
\mu_{n-f}^{l_{b, a}}=\mu_{n-f(b)-\mu_{n-f}(a)}^{\mu_{n-f}} \mu_{n-f} \mu_{n}^{-\left(\mu_{n-f}(b)-\mu_{n-f}(a)\right)}=\mu_{\mu_{n-f}(b)-\mu_{n-f}(a)},
$$

where the index $\mu_{n-f}(b)-\mu_{n-f}(a)$ is read modulo $n-f$. Since $1 \leqslant l_{b, a}<n-f$, then $\mu_{n-f}^{l_{b, a}}$ and $\mu_{n-f}$ have the same set of fixed points. Thus, the equalities above imply that, modulo $n-f, \mu_{n-f}(b)-\mu_{n-f}(a) \in F_{n-f}$.

Corollary 37. $\mu_{n-f}\left(F_{n}\right)=F_{k}$, for some $k \notin F_{n} \cup F_{n-f}$.

Proof. Let $a \in F_{n}$ and let $\mu_{n-f}(a)=k$, where $k \notin F_{n} \cup F_{n-f}$ by Lemma 29. For each $b \in F_{n} \backslash\{a\}$, we have that $\mu_{n-f}(b)-\mu_{n-f}(a) \in F_{n-f}$ by Lemma 36 . Therefore, by Corollary $35, \mu_{n-f}\left(F_{n}\right) \subset F_{k}$. Since $\left|F_{n}\right|=\left|F_{k}\right|$ and $\mu_{n-f}$ is a bijection, $\mu_{n-f}\left(F_{n}\right)=F_{k}$.

Corollary 38. Let $\mu_{n-f}\left(F_{n}\right)=F_{k}, k \notin F_{n} \cup F_{n-f}$, see Corollary 37. Then $\mu_{i\left(\frac{n-f}{f}\right)}\left(F_{n}\right)=$ $F_{k}, \forall i \in\{1, \ldots, f\}$.

Proof. Given $a \in F_{n}$ and $i \in\{1, \ldots, f\}$, we prove that $\mu_{i\left(\frac{n-f}{f}\right)}(a) \in F_{k}$. We have

$$
\begin{aligned}
\mu_{i\left(\frac{n-f}{f}\right)}(a) & =\mu_{n}^{i\left(\frac{n-f}{f}\right)} \mu_{n-f} \mu_{n}^{-i\left(\frac{n-f}{f}\right)}(a)=\mu_{n}^{i\left(\frac{n-f}{f}\right)} \mu_{n-f}(a)= \\
& =\mu_{n-f}(a)+i\left(\frac{n-f}{f}\right) \in F_{\mu_{n-f}(a)}=F_{k} .
\end{aligned}
$$

by Corollary 35. Hence, $\mu_{i\left(\frac{n-f}{f}\right)}\left(F_{n}\right) \subset F_{k}$. As $\left|F_{n}\right|=\left|F_{k}\right|$ and $\mu_{i\left(\frac{n-f}{f}\right)}$ is a bijection, $\mu_{i\left(\frac{n-f}{f}\right)}\left(F_{n}\right)=F_{k}$ and the result follows.

Corollary 39. $\mu_{i\left(\frac{n-f}{f}\right)} \neq \mu_{j\left(\frac{n-f}{f}\right)}$, where $1 \leqslant i \neq j \leqslant f$.

Proof. Given $a \in F_{n}, \mu_{i\left(\frac{n-f}{f}\right)}(a)=\mu_{n-f}(a)+i\left(\frac{n-f}{f}\right), \forall i \in\{1, \ldots, f\}$. Then, given $i, j \in\{1, \ldots, f\}$, with $i \neq j, \mu_{i\left(\frac{n-f}{f}\right)} \neq \mu_{j\left(\frac{n-f}{f}\right)}$.

With the previous results, we are now able to prove a very important proposition. In fact, this proposition is used in the following section to prove that there are no quandles of cyclic type of order $n$ with $f$ fixed points such that $n=3 f$ for $f>2$.

Before presenting the proposition, we just recall some of the terminology we used in assertion 2. in Theorem 28. 
Definition 40. Let $h$ and $h^{\prime}$ be associate indices. We let $l_{h, h^{\prime}}$ denote the positive integer such that $\mu_{h}=\mu_{h^{\prime}}^{l_{h, h^{\prime}}}$, where $G C D\left(n-f, l_{h, h^{\prime}}\right)=1,1 \leqslant l_{h, h^{\prime}}<n-f$, in accordance with assertion 2. in Theorem 28. Moreover, since the associate indices to $n-f$ are $i\left(\frac{n-f}{f}\right)$,

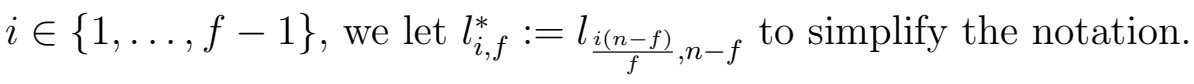

Proposition 41. $\left\{l_{i, f}^{*}: 1 \leqslant i \leqslant f\right\}=\left\{1+j\left(\frac{n-f}{f}\right): 0 \leqslant j<f\right\}$

Proof. By Corollary 37, there exists an index $k \notin F_{n} \cup F_{n-f}$ such that $\mu_{n-f}\left(F_{n}\right)=F_{k}$. Moreover, we write $F_{k}=\left\{g_{k}^{1}, \ldots, g_{k}^{f}\right\}$, where the indices $g_{k}^{i}$ are labelled in such a way that there exist positive integers $l_{i}>1, \forall i \in\{1, \ldots, f\}$, such that $\mu_{n-f}^{l_{i}}\left(g_{k}^{i-1}\right)=g_{k}^{i}$ and $\mu_{n-f}^{m}\left(g_{k}^{i-1}\right) \notin F_{k}$ for $1<m<l_{i}$, where we take $g_{k}^{0}:=g_{k}^{f}$. Basically, each $l_{i}$ is the smallest positive integer such that $\mu_{n-f}^{l_{i}}\left(g_{k}^{i-1}\right) \in F_{k}$, and we have $\mu_{n-f}^{l_{i}}\left(g_{k}^{i-1}\right)=g_{k}^{i}$. We note that the indices in $F_{k}$ belong to the non-singular cycle of $\mu_{n-f}$ and we prove the following lemma.

Lemma 42. The indices in $F_{k}$ are equally spaced in the non-singular cycle of $\mu_{n-f}$. In particular, the $l_{i}$ 's referred to above satisfy $l_{i}=(n-f) / f, \forall i \in\{1, \ldots, f\}$.

Proof. Suppose this is not true. Then, there is an index $j_{0} \in\{1, \ldots, f\}$ such that $l_{j_{0}}<l_{j_{0}^{\prime}}$, for a certain $j_{0}^{\prime} \in\{1, \ldots, f\}$. Assume, without loss of generality, that the indices in $F_{n}=\left\{g_{n}^{1}, \ldots, g_{n}^{f}\right\}$ are labelled in such a way that $\mu_{n-f}\left(g_{n}^{i}\right)=g_{k}^{i}, \forall i \in\{1, \ldots, f\}$. Taking $g_{n}^{0}:=g_{n}^{f}$,

$$
\mu_{n-f}^{l_{j_{0}}}\left(g_{k}^{j_{0}-1}\right)=g_{k}^{j_{0}} \Leftrightarrow \mu_{n-f}^{l_{j_{0}}}\left(\mu_{n-f}\left(g_{n}^{j_{0}-1}\right)\right)=g_{k}^{j_{0}} \Leftrightarrow \mu_{n-f}^{l_{j_{0}}+1}\left(g_{n}^{j_{0}-1}\right)=g_{k}^{j_{0}} .
$$

By Corollary $38, \mu_{n-f}$ and its associate permutations are bijections from $F_{n}$ to $F_{k}$, where $\left|F_{k}\right|=f$. These $f$ permutations are of the form $\mu_{i\left(\frac{n-f}{f}\right)}=\mu_{n-f}^{l_{i, f}^{*}}$, with $i \in$ $\{1, \ldots, f\}$ and $l_{f, f}^{*}=1$, and they are all different from each other by Corollary 39. Hence, any two of them have different images at $g_{n}^{j_{0}-1}$, otherwise at least two of these permutations would be equal to each other, conflicting with Corollary 39. (in particular, $\left\{l_{i, f}^{*}: 1 \leqslant\right.$ $i \leqslant f\}$ has exactly $f$ elements). Then, there has to be an integer $j_{1} \in\{1, \ldots, f\}$ such that $\mu_{j_{1}\left(\frac{n-f}{f}\right)}\left(g_{n}^{j_{0}-1}\right)=\mu_{n-f}^{l_{j_{1}, f}^{*}}\left(g_{n}^{j_{0}-1}\right)=g_{k}^{j_{0}}$. Hence, comparing with $(3), l_{j_{1}, f}^{*}=l_{j_{0}}+1$ and $\mu_{j_{1}\left(\frac{n-f}{f}\right)}=\mu_{n-f}^{l_{j_{0}}+1}$, and thus

$$
\mu_{j_{1}\left(\frac{n-f}{f}\right)}\left(g_{n}^{j_{0}^{\prime}-1}\right)=\mu_{n-f}^{l_{j_{0}}+1}\left(g_{n}^{j_{0}^{\prime}-1}\right)=\mu_{n-f}^{l_{j_{0}}}\left(\mu_{n-f}\left(g_{n}^{j_{0}^{\prime}-1}\right)\right)=\mu_{n-f}^{l_{j_{0}}}\left(g_{k}^{j_{0}^{\prime}-1}\right) .
$$

However, $\mu_{n-f}^{l_{j_{0}}}\left(g_{k}^{j_{0}^{\prime}-1}\right) \notin F_{k}$, as $1<l_{j_{0}}<l_{j_{0}^{\prime}}$. This is a contradiction, since $\mu_{j_{0}\left(\frac{n-f}{f}\right)}\left(F_{n}\right)=$ $F_{k}$. Thus, the indices in $F_{k}$ are equally spaced in the cycle of length $n-f$ of $\mu_{n-f}$. 
We now resume the proof of Proposition 41. Given $g_{k}^{j} \in F_{k}$, there is an index $g_{n}^{j} \in F_{n}$ such that $\mu_{n-f}\left(g_{n}^{j}\right)=g_{k}^{j}$. Then, $\forall i \in\{1, \ldots, f\}$,

$$
F_{k} \ni \mu_{i\left(\frac{n-f}{f}\right)}\left(g_{n}^{j}\right)=\mu_{n-f}^{l_{i, f}^{*}}\left(g_{n}^{j}\right)=\mu_{n-f}^{l_{i, f}^{*}-1}\left(\mu_{n-f}\left(g_{n}^{j}\right)\right)=\mu_{n-f}^{l_{i, f}^{*}-1}\left(g_{k}^{j}\right)
$$

that is, $\forall i \in\{1, \ldots, f\}$, we have $\mu_{n-f}^{l_{i, f}^{*}-1}\left(F_{k}\right) \in F_{k}$. Since the $f$ indices in $F_{k}$ are equally spaced in the cycle of length $n-f$ of $\mu_{n-f}, f\left(l_{i, f}^{*}-1\right)$ has to be a multiple of $n-f$, that is, $f\left(l_{i, f}^{*}-1\right)=k_{i}(n-f)$, for some natural number $0 \leqslant k_{i}<f, \forall i \in\{1, \ldots, f\}$. This is equivalent to saying that

$$
f\left(l_{i, f}^{*}-1\right)=k_{i}(n-f) \Leftrightarrow l_{i, f}^{*}-1=k_{i}\left(\frac{n-f}{f}\right) \Leftrightarrow l_{i, f}^{*}=1+k_{i}\left(\frac{n-f}{f}\right),
$$

for some natural number $0 \leqslant k_{i}<f, \forall i \in\{1, \ldots, f\}$. Hence, the set $\left\{l_{i, f}^{*}: 1 \leqslant i \leqslant f\right\}$ is contained in $\left\{1+j\left(\frac{n-f}{f}\right): 0 \leqslant j<f\right\}$. Since these two sets must have the same cardinality, the result follows.

Remark 43. We remark that the integers in $\left\{l_{i, f}^{*}: 1 \leqslant i \leqslant f\right\}$ still have to verify the conditions presented in assertion 2. in Theorem 28, that is, $G C D\left(n-f, l_{i, f}^{*}\right)=1,1 \leqslant$ $l_{i, f}^{*}<n-f, \forall i \in\{1, \ldots, f\}$. In fact, there are certain pairs of integers $(n, f)$ for which these conditions are not satisfied if $l_{i, f}^{*}$ has the form $1+\frac{i(n-f)}{f}$ with $0 \leqslant i<f$, where $l_{0, f}^{*}:=l_{f, f}^{*}$. Thus the corresponding quandles of cyclic type of order $n$ with $f$ fixed points cannot exist. For example, there cannot be quandles of cyclic type of order 28 with 7 fixed points, but there can be quandles of cyclic type of order 6 with 2 fixed points. Therefore, from now on, we are only working with pairs of integers $(n, f)$ for which these conditions are satisfied.

\subsection{Quandles of Cyclic Type of Order $n$ with $f$ Fixed Points such that $n=3 f$}

In this Subsection, we show there are no quandles of cyclic type of order $n$ with $f$ fixed points such that $n=3 f$ for $f>2$. We also prove that the only quandle of cyclic type of order 6 with 2 fixed points, up to isomorphism, is $Q_{6}^{2}$.

First, we prove a result which is a direct consequence of Proposition 41.

Corollary 44. Given a quandle of cyclic type $Q$ of order $n$ with $f$ fixed points such that $n=3 f, \mu_{n-f}^{-1}=\mu_{2 i}$, for a certain integer $i \in\{1, \ldots, f\}$.

Proof. By Proposition 41, $\left\{l_{i, f}^{*}: 1 \leqslant i \leqslant f\right\}=\left\{1+j\left(\frac{n-f}{f}\right): 0 \leqslant j<f\right\}$. Taking $j=$ $f-1$, we conclude there exists an integer $i^{\prime} \in\{1, \ldots, f\}$ such that $l_{i^{\prime}, f}^{*}=1+(f-1)\left(\frac{n-f}{f}\right)$. As $n=3 f$, we get

$$
l_{i^{\prime}, f}^{*}=l_{\frac{i^{\prime}(n-f)}{f}, n-f}=l_{2 i^{\prime}, 2 f}=1+2(f-1)=2 f-1=(n-f)-1 \equiv-1 \bmod n-f,
$$

that is, there exists an integer $i^{\prime} \in\{1, \ldots, f\}$ such that $\mu_{n-f}^{-1}=\mu_{2 f}^{-1}=\mu_{2 i^{\prime}}$. 
Corollary 44 tells us that, for any quandle of cyclic type $Q$ of order $n$ with $f$ fixed points such that $n=3 f, \mu_{n-f}^{-1}$ is a permutation of $Q$ and it is of the form $\mu_{n-f}^{-1}=\mu_{2 f}^{-1}=\mu_{2 i}$, for a certain integer $i \in\{1, \ldots, f\}$. We now prove a lemma, similar to Lemma 36, which also has some very useful consequences.

Lemma 45. Given distinct indices $a, b \in F_{n}, \mu_{n-f}^{-1}(b)-\mu_{n-f}^{-1}(a) \in F_{n-f}$, where this index is read modulo $n-f$.

Proof. Let $a, b \in F_{n}$. By assertion 2. in Theorem 28, we have $\mu_{b}=\mu_{a}^{l_{b, a}}$. Hence, using assertion 5. in Theorem 28,

$$
\begin{gathered}
\mu_{n}^{\mu_{n-f}^{-1}(a)} \mu_{n-f}^{l_{b, a}} \mu_{n}^{-\mu_{n-f}^{-1}(a)}=\left(\mu_{n-f}^{\mu_{n-f}^{-1}(a)} \mu_{n-f} \mu_{n}^{-\mu_{n-f}^{-1}(a)}\right)^{l_{b, a}}=\left(\mu_{n-f}^{-1} \mu_{a} \mu_{n-f}\right)^{l_{b, a}}= \\
=\mu_{n-f}^{-1} \mu_{a}^{l_{b, a}} \mu_{n-f}=\mu_{n-f}^{-1} \mu_{b} \mu_{n-f}=\mu_{n}^{\mu_{n-f}^{-1}(b)} \mu_{n-f} \mu_{n}^{-\mu_{n-f}^{-1}(b)},
\end{gathered}
$$

which implies, by assertion 3. in Theorem 28, that

$$
\mu_{n-f}^{l_{b, a}}=\mu_{n-f}^{\mu_{n-f}^{-1}(b)-\mu_{n-f}^{-1}(a)} \mu_{n-f} \mu_{n}^{-\left(\mu_{n-f}^{-1}(b)-\mu_{n-f}^{-1}(a)\right)}=\mu_{\mu_{n-f}^{-1}(b)-\mu_{n-f}^{-1}(a)},
$$

where the index $\mu_{n-f}^{-1}(b)-\mu_{n-f}^{-1}(a)$ is read modulo $n-f$. Since $1 \leqslant l_{b, a}<n-f$, then $\mu_{n-f}^{l_{b, a}}$ and $\mu_{n-f}$ have the same set of fixed points. Thus, the equalities above imply that, modulo $n-f, \mu_{n-f}^{-1}(b)-\mu_{n-f}^{-1}(a) \in F_{n-f}$.

Corollary 46. Given $a, b \in F_{n}, \mu_{n-f}(b)-\mu_{n-f}(a)=\mu_{n-f}^{-1}(b)-\mu_{n-f}^{-1}(a)$, where these two indices are read modulo $n-f$.

Proof. Any two permutations from $\mu_{1}$ to $\mu_{n-f}$ are distinct. Indeed, given an index $a \in F_{n}$ and for each index $k \in\{1, \ldots, n-f\}$, we have, by assertion 3. in Theorem 28,

$$
\mu_{k}(a)=\mu_{n}^{k} \mu_{n-f} \mu_{n}^{-k}(a)=\mu_{n}^{k} \mu_{n-f}(a)=\mu_{n-f}(a)+k .
$$

Since we also have, by Lemmas 36 and 45, that

$$
\mu_{\mu_{n-f}(b)-\mu_{n-f}(a)}=\mu_{n-f}^{l_{a, b}}=\mu_{\mu_{n-f}^{-1}(b)-\mu_{n-f}^{-1}(a)},
$$

where the indices $\mu_{n-f}(b)-\mu_{n-f}(a)$ and $\mu_{n-f}^{-1}(b)-\mu_{n-f}^{-1}(a)$ are read modulo $n-f$, we get

$$
\mu_{n-f}(b)-\mu_{n-f}(a)=\mu_{n-f}^{-1}(b)-\mu_{n-f}^{-1}(a),
$$

where these two indices are read modulo $n-f$.

We now have all the results we need to prove there are no quandles of cyclic type of order $n$ with $f$ fixed points such that $n=3 f$ for $f>2$. This is the result we state in the following proposition. 
Proposition 47. There are no quandles of cyclic type of order $n$ with $f$ fixed points such that $n=3 f$ for $f>2$.

Proof. Suppose $Q$ is a quandle of cyclic type of order $n$ with $f$ fixed points such that $n=3 f$. First of all, we note that $Q=F_{1} \cup F_{n-f} \cup F_{n}$. We know $\mu_{n-f}\left(F_{n-f}\right)=F_{n-f}$ and we have $\mu_{n-f}\left(F_{n}\right)=F_{1}$ by Corollary 44 . Since $\mu_{n-f}$ is a bijection, we have $\mu_{n-f}\left(F_{1}\right)=F_{n}$. Now, let $a \in F_{n}$ and suppose $\mu_{n-f}(a)=j$ and $\mu_{n-f}^{-1}(a)=k$, where $j, k \in F_{1}$. We note that $j \neq k$, otherwise $\mu_{n-f}$ would have a cycle of length 2 . This is not possible as the length of this cycle is $n-f=3 f-f=2 f \geqslant 4$. For each $i \in\{1, \ldots, n-f\}$, we have by assertion 3. in Theorem 28, reading the indices modulo $n-f$,

$$
\mu_{i}(a)=\mu_{n}^{i} \mu_{n-f} \mu_{n}^{-i}(a)=\mu_{n}^{i} \mu_{n-f}(a)=\mu_{n}^{i}(j)=j+i .
$$

and, again by assertion 3. in Theorem 28, reading the indices modulo $n-f$,

$$
\mu_{i}(k+i)=\mu_{n}^{i} \mu_{n-f} \mu_{n}^{-i}(k+i)=\mu_{n}^{i} \mu_{n-f}(k)=\mu_{n}^{i}(a)=a,
$$

By Corollary 44, we know that there is an index $i^{\prime} \in\{1, \ldots, f\}$ such that $\mu_{2 i^{\prime}}=\mu_{n-f}^{-1}$. Therefore, we have that $\mu_{2 i^{\prime}}(a)=k$ and $\mu_{2 i^{\prime}}(j)=a$. Since $\mu_{2 i^{\prime}}(a)=j+2 i^{\prime}$ by (4), we conclude that $k=j+2 i^{\prime}$. Hence, $\mu_{2 i^{\prime}}(j)=\mu_{2 i^{\prime}}\left(k-2 i^{\prime}\right)$ and we also conclude $\mu_{2 i^{\prime}}(k-$ $\left.2 i^{\prime}\right)=a$. But $\mu_{2 i^{\prime}}\left(k+2 i^{\prime}\right)=a$ by (5), which means that we have either $2 i^{\prime}=n-f$ or $2 i^{\prime}=(n-f) / 2$. Since $2 i^{\prime}$ cannot be equal to $n-f$, as this forces $k$ to be equal to $j$, we then have $2 i^{\prime}=(n-f) / 2$. Now, suppose $\mu_{n-f}(j)=b \in F_{n}$, with $b \neq a$. Noting that we have $\mu_{n-f}(a)-\mu_{n-f}(b)=\mu_{n-f}^{-1}(a)-\mu_{n-f}^{-1}(b)$ by Corollary 46 , we get

$$
\mu_{n-f}^{-1}(b)-\mu_{n-f}(b)=\mu_{n-f}^{-1}(a)-\mu_{n-f}(a)=k-j=\frac{n-f}{2} .
$$

Since $\mu_{n-f}^{-1}(b)=j$, this forces $\mu_{n-f}(b)$ to be equal to $k$. Therefore, $\mu_{n-f}$ has a cycle $\tau$ of length 4 , where $\tau=(a j b k)$. Hence, for any quandle of cyclic type of order $n$ with $f$ fixed points such that $n=3 f, f=2$ and $n=6$ and, thus, there are no quandles of cyclic type of order $n$ with $f$ fixed points such that $n=3 f$ for $f>2$.

Corollary 48. There is only one quandle of cyclic type of order 6 with 2 fixed points, up to isomorphism.

Proof. $\mu_{6}=\left(\begin{array}{lll}1 & 2 & 3\end{array}\right)(5)(6)$, up to isomorphism, by assertion 1. in Theorem 28 and then $F_{5}=\{5,6\}=F_{6} . F_{2}=\{2,4\}=F_{4}$ by Proposition 34 , and so $F_{1}=\{1,3\}=F_{3}$. Now, by Corollary 37, we can either have $\mu_{4}=(2)(4)(1635)$ or $\mu_{4}=(2)(4)(1536)$. However, straightforward calculations show that the latter does not satisfy assertion 4. in Theorem 28, and hence $\mu_{4}=(2)(4)\left(\begin{array}{l}1 \\ 6\end{array} 3\right.$ 5). By Theorem 28, we can write $\mu_{1}, \mu_{2}, \mu_{3}$ and $\mu_{5}$ as functions of $\mu_{6}$ and $\mu_{4}$, thus $\mu_{4}$ determines one single quandle, which is precisely $Q_{6}^{2}$. Its multiplication table is displayed in Table 1. 


\subsection{Quandles of Cyclic Type of Order $n$ with $f$ Fixed Points such that $n>3 f$}

In this Subsection, we use the results from the previous Subsections to show that there are no quandles of cyclic type of order $n$ with $f$ fixed points such that $n=c f$ for $c>3$. This is the result we state in the following proposition, where in its proof the free indices are read modulo $n-f$.

Proposition 49. There are no quandles of cyclic type of order $n$ with $f$ fixed points such that $n=c f$, for $c>3$.

Proof. Let $\mu_{n-f}\left(F_{n}\right)=F_{k}$, in accordance with Corollary 37. We know the indices in $F_{k}$ belong to the cycle of length $n-f$ of $\mu_{n-f}$, they are all equally spaced in this cycle by Lemma 42 and their inverse images are in $F_{n}$. Therefore, the indices in $F_{n}$ are also equally spaced in the cycle of length $n-f$ of $\mu_{n-f}$. Now, we have that for each $m \in$ $\{1, \ldots, n-f\} \backslash\left\{\mu_{n-f}^{-1}(n-f+1), \ldots, \mu_{n-f}^{-1}(n)\right\}$, there is an integer $1 \leqslant k_{m}<n-f$ such that $\mu_{n}^{-\mu_{n-f}(m)} \mu_{n-f} \mu_{n}^{m}=\sigma \tau^{k_{m}}$, by assertion 6 . in Theorem 28. We now prove that each $m$ gives rise to a different $k_{m}$. In fact, given $a \in F_{n}$,

$$
\tau^{k_{m}}(a)=\sigma \tau^{k_{m}}(a)=\mu_{n-f}^{k_{m}}(a)=\mu_{n}^{-\mu_{n-f}(m)} \mu_{n-f} \mu_{n}^{m}(a)=\mu_{n-f}(a)-\mu_{n-f}(m),
$$

which has a different value for each $\left.m \in\{1, \ldots, n-f\} \backslash\left\{\mu_{n-f}^{-1}(n-f+1), \ldots, \mu_{n-f}^{-1}(n)\right\}\right)$. Therefore, assertion 6 . in Theorem 28 provides us with a total of $n-2 f$ different equalities and, in particular, $n-2 f$ different integers $k_{m}$. Now, given $a, b \in F_{n}$, we can combine the two equalities

$$
\begin{aligned}
& \mu_{n-f}(a)-\mu_{n-f}(m)=\mu_{n-f}^{k_{m}}(a), \\
& \mu_{n-f}(b)-\mu_{n-f}(m)=\mu_{n-f}^{k_{m}}(b),
\end{aligned}
$$

in order to get

$$
F_{n-f} \ni \mu_{n-f}(a)-\mu_{n-f}(b)=\mu_{n-f}^{k_{m}}(a)-\mu_{n-f}^{k_{m}}(b) .
$$

Indeed, we know exactly what are the $n-2 f$ different integers $k_{m}$ which satisfy this equality. Suppose that $k_{m} \in\left\{i\left(\frac{n-f}{f}\right): 1 \leqslant i \leqslant f\right\}=F_{n-f}$. Therefore, $\mu_{n-f}^{k_{m}}(a), \mu_{n-f}^{k_{m}}(b) \in F_{n}$ by Lemma 42 and $0<\left|\mu_{n-f}^{k_{m}}(a)-\mu_{n-f}^{k_{m}}(b)\right|<f$, since $F_{n}=\{n-f+1, \ldots, n\}$. However, we can choose indices $a, b \in F_{n}$ such that $f \leqslant\left|\mu_{n-f}(a)-\mu_{n-f}(b)\right|$, which is a contradiction. If $f$ is even, for example, pick $a, b \in F_{n}$ such that $\mu_{n-f}(b)=\mu_{n-f}(a)+\frac{f}{2}\left(\frac{n-f}{f}\right)$. If $f$ is odd, pick $a, b \in F_{n}$ such that $\mu_{n-f}(b)=\mu_{n-f}(a)+\frac{f \pm 1}{2}\left(\frac{n-f}{f}\right)$. Then, $k_{m} \in\{1, \ldots, n-f\} \backslash F_{n-f}$, and this set has exactly $n-2 f$ elements. Now, given a quandle of cyclic type of order $n$ with $f$ fixed points such that $n=c f$, where $c>3$, we know $2 \in\{1, \ldots, n-f\} \backslash F_{n-f}$. Hence, given $a, b \in F_{n}$,

$$
\mu_{n-f}(a)-\mu_{n-f}(b)=\mu_{n-f}^{2}(a)-\mu_{n-f}^{2}(b) .
$$


However, we also have, by assertion 3. in Theorem 28, that

$$
\begin{aligned}
& \mu_{\mu_{n-f}(b)-\mu_{n-f}(a)}\left(\mu_{n-f}(b)\right)=\mu_{n}^{\mu_{n-f}(b)-\mu_{n-f}(a)} \mu_{n-f} \mu_{n}^{-\left(\mu_{n-f}(b)-\mu_{n-f}(a)\right)}\left(\mu_{n-f}(b)\right)= \\
& =\mu_{n-f}^{\mu_{n-f}(b)-\mu_{n-f}(a)} \mu_{n-f}\left(\mu_{n-f}(a)\right)=\mu_{n-f}^{2}(a)-\mu_{n-f}(a)+\mu_{n-f}(b)=\mu_{n-f}^{2}(b)= \\
& =\mu_{n-f}\left(\mu_{n-f}(b)\right),
\end{aligned}
$$

implying that the two associate permutations $\mu_{\mu_{n-f}(b)-\mu_{n-f}(a)}$ and $\mu_{n-f}$ (see Proposition 34 and Corollary 35) have the same image at a point that is not a fixed point of these permutations, as $\mu_{n-f}(b) \notin F_{n-f}$. Hence, these permutations must be equal to each other, which is a contradiction, as these permutations are different from each other by Corollary 39. Therefore, there are no quandles of cyclic type of order $n$ with $f$ fixed points such that $n=c f$, for $c>3$, and the result follows.

\subsection{Classifying Quandles of Cyclic Type of Order $n$ with $f$ Fixed Points such that $n>2 f$}

In this Subsection, we prove Assertion 1.(b) in Theorem 14 and make a few observations regarding $Q_{6}^{2}$.

Proof. (Assertion 1.(b) in Theorem 14) Immediate from Corollary 22, Propositions 47 and 49 and Corollary 48.

Example 50. $Q_{6}^{2}$, whose multiplication table is displayed in Table 1, is the only quandle of cyclic type of order $n$ with $f$ fixed points such that $n>2 f$, up to isomorphism. In particular, $Q_{6}^{2}$ is not a simple quandle (since it admits a non-trivial congruence). Indeed, by Proposition 23, for $n \geqslant 2 f$, "i is associate to j" generates an equivalence relation on $Q_{6}^{2}$, which is also a congruence relation on this set, as it respects the binary operation of the quandle. In Table 4, we see the quotient of $Q_{6}^{2}$ by this congruence relation, which we denote by $\sim$. This quotient is clearly isomorphic to $R_{3}$, since "the product" of any two distinct elements equals the other element.

\begin{tabular}{|c|ccc|}
\hline $\bar{*}$ & $\{1,3\}$ & $\{2,4\}$ & $\{5,6\}$ \\
\hline$\{1,3\}$ & $\{1,3\}$ & $\{5,6\}$ & $\{2,4\}$ \\
$\{2,4\}$ & $\{5,6\}$ & $\{2,4\}$ & $\{1,3\}$ \\
$\{5,6\}$ & $\{2,4\}$ & $\{1,3\}$ & $\{5,6\}$ \\
\hline
\end{tabular}

Table 4: $Q_{6}^{2} / \sim$ multiplication table.

\section{Families of Quandles of Cyclic Type of Order $n$ with $f$ Fixed Points in the Range $f+2 \leqslant n \leqslant 2 f$}

Theorem 51. Let $f$ be an integer strictly greater than 1 and $n$ a positive integer such that $f+2 \leqslant n \leqslant 2 f$. Assume further that $(n-f) \mid f$. For each $i$ such that $1 \leqslant i \leqslant \frac{n}{n-f}$, 
consider the permutations of $\{1,2, \ldots, n\}$, given by

$$
\begin{aligned}
\mu_{(i-1)(n-f)+1} & =\mu_{(i-1)(n-f)+1}=\cdots=\mu_{(i-1)(n-f)+1}= \\
& =(i(n-f)+1 i(n-f)+2 \cdots(i+1)(n-f)) .
\end{aligned}
$$

This sequence of permutations defines a quandle of cyclic type of order $n$ with $f$ fixed points over the set $\{1,2, \ldots, n\}$.

Proof. The proof of this Theorem is basically a rearrangement of the argument for the proof of the first statement of Corollary 31, the existence of a quandle of cyclic type of order $2 f$ with $f$ fixed points. We add it here for completeness.

Let

$(i-1)(n-f)+1 \leqslant j, j^{\prime} \leqslant i(n-f) \quad$ and $\quad\left(i^{\prime}-1\right)(n-f)+1 \leqslant k \leqslant i^{\prime}(n-f) \quad\left(i \neq i^{\prime}\right)$.

Then,

$$
\mu_{j}(k)=k+1 \Longrightarrow \mu_{k+1}=\mu_{j} \mu_{k} \mu_{j}^{-1}=\mu_{k} \quad \text { since } \mu_{j} \text { and } \mu_{k} \text { commute . }
$$

Also,

$$
\mu_{j}\left(j^{\prime}\right)=j^{\prime} \Longrightarrow \mu_{j^{\prime}}=\mu_{j} \mu_{j^{\prime}} \mu_{j}^{-1}=\mu_{j^{\prime}} \quad \text { since } \mu_{j} \text { and } \mu_{j^{\prime}} \text { are equal . }
$$

This completes the proof.

\subsection{Extracting - Adjoining a Common Fixed Point}

Definition 52. Let $Q$ be a quandle of cyclic type with several fixed points. If $g_{0} \in Q$ is such that it is a fixed point for any of the permutations of $Q, g_{0}$ is called a common fixed point of $Q$.

Example 53. In Table 5 we provide the multiplication table of a quandle of cyclic type of order 5 and 3 fixed points. The order and number of fixed points of this quandle satisfy $f+2 \leqslant n \leqslant 2 f$. Moreover, its permutations are

$$
\mu_{1}=(1)(2)(3)(45)=\mu_{2}=\mu_{3} \quad \mu_{5}=(1)(4)(5)(23)=\mu_{4} .
$$

Thus, 1 is a common fixed point for this quandle.

\begin{tabular}{|c|ccccc|}
\hline$*$ & 1 & 2 & 3 & 4 & 5 \\
\hline 1 & 1 & 1 & 1 & 1 & 1 \\
2 & 2 & 2 & 2 & 3 & 3 \\
3 & 3 & 3 & 3 & 2 & 2 \\
4 & 5 & 5 & 5 & 4 & 4 \\
5 & 4 & 4 & 4 & 5 & 5 \\
\hline
\end{tabular}

Table 5: Quandle of cyclic type of order 5 and 3 fixed points with a common fixed point: 1. See [6], page 176 . 
The next two Theorems show us when we can extract a common fixed point (Theorem 16) and when we can adjoin a common fixed point (Theorem 17). We repeat their statements here for completeness. Once Theorem 17 is proved, combining it with Theorem 51 and iterating the procedure, provides an infinite sequence of quandles of cyclic type with several fixed points within the range $f+2 \leqslant n \leqslant 2 f$ where $n$ is the order and $f$ the number of fixed points. This is the content of Corollary 18.

Theorem 54. Suppose $f$ is an integer strictly greater than 2 and $n$ a positive integer such that $f+2 \leqslant n \leqslant 2 f$. Consider a quandle of cyclic type of order $n$ and $f$ fixed points over the set $Q=\{1,2, \ldots, n\}$ with sequence of permutations $\mu_{i}$ with $i \in\{1,2, \ldots, n\}$. Assume further $g_{0} \in Q$ is a common fixed point of $Q$. Then, the set $Q^{\prime}=Q \backslash\left\{g_{0}\right\}$ along with the sequence of permutations $\mu_{i}^{\prime}=\left.\mu_{i}\right|_{Q^{\prime}}$ for each $i \in Q^{\prime}$ defines a quandle of cyclic type of order $n-1$ with $f-1$ fixed points. We call this the extraction of the common fixed point $g_{0}$.

Proof. We keep the notation and terminology from the statement. Since $\mu_{i}\left(g_{0}\right)=g_{0}$, for each $i \in Q$, then

$$
\mu_{g_{0}}=\mu_{i} \mu_{g_{0}} \mu_{i}^{-1} \Longleftrightarrow \mu_{i} \mu_{g_{0}}=\mu_{g_{0}} \mu_{i},
$$

which amounts to saying that

$$
\mu_{g_{0}}=\mu_{i}^{k_{i}} \quad\left(\text { for some } i \in Q, \text { for some } k_{i} \in \mathbf{Z}\right) \quad \text { OR } \quad C_{g_{0}} \cap C_{i}=\emptyset \text {. }
$$

Assume

$$
\mu_{g_{0}}=\left(\begin{array}{llll}
g_{1} & g_{2} & \ldots & g_{n-f}
\end{array}\right)
$$

Then,

$$
g_{i+1}=\mu_{g_{0}}\left(g_{i}\right) \Longrightarrow \mu_{g_{i+1}}=\mu_{g_{0}} \mu_{g_{i}} \mu_{g_{0}}^{-1}=\mu_{g_{i}} .
$$

So the associate permutations to the permutations corresponding to the elements moved by $\mu_{g_{0}}$, are all equal to one another.

Consider now the set $Q^{\prime}=Q \backslash\left\{g_{0}\right\}$ along with the sequence of permutations $\mu_{i}^{\prime}=\left.\mu_{i}\right|_{Q^{\prime}}$ for each $i \in Q^{\prime}$. For each $i, j \in Q^{\prime}$, we have

$$
\mu_{\mu_{i}(j)}=\mu_{i} \mu_{j} \mu_{i}^{-1} \Longleftrightarrow \mu_{\mu_{i}^{\prime}(j)}^{\prime}=\mu_{i}^{\prime} \mu_{j}^{\prime} \mu_{i}^{\prime-1},
$$

which completes the proof.

Theorem 55. Let $n$ be an integer greater than 2. Let $Q$ be the underlying set of a quandle whose permutations are denoted $\mu_{i}$, for each $i \in Q$. Let $g_{0} \notin Q$ and consider the set $Q^{\prime}=Q \cup\left\{g_{0}\right\}$. Suppose there is a permutation, $\mu$, of the elements of $Q$, such that $\mu \mu_{i}=\mu_{i} \mu$, for each $i \in Q$. Then, $Q^{\prime}$ along with the permutations

$$
\mu_{i}^{\prime}=\left(g_{0}\right) \mu_{i} \quad \text { for each } i \in Q \quad \text { and } \quad \mu_{g_{0}}^{\prime}=\left(g_{0}\right) \mu
$$

is a quandle with a common fixed point, $g_{0}$.

Proof. For each $i, i^{\prime} \in Q, \mu_{\mu_{i}\left(i^{\prime}\right)}=\mu_{i} \mu_{i^{\prime}} \mu_{i}^{-1} \Longleftrightarrow \mu_{\mu_{i}^{\prime}\left(i^{\prime}\right)}^{\prime}=\mu_{i}^{\prime} \mu_{i^{\prime}}^{\prime} \mu_{i}^{\prime-1}$ and $\mu \mu_{i}=\mu_{i} \mu \Longleftrightarrow$ $\mu^{\prime} \mu_{i}^{\prime}=\mu_{i}^{\prime} \mu^{\prime}$. This completes the proof. 


\section{$5 \quad$ Further Research}

In this article we looked into the classification of quandles of cyclic type of order $n$ with $f$ fixed points. We realize that these quandles split into three sorts according to the ranges their $(n, f)$ 's lie in. If $n>2 f$, then these quandles are connected. As a matter of fact, there is only one such quandle which occurs for $n=6$ and $f=2$; it is the octahedron quandle. For each integer $f>2$, there is exactly one such quandle of order $n=2 f$ and it is not connected. Finally, in the range $2<f+1<n<2 f$, such quandles are not connected and there seem to be plenty of them.

With the techniques developed in this article, we plan on looking into the classification of other families of quandles like those with constant profile with $f$ fixed points and two non-singular cycles, to begin with. We also plan on taking a fresh look at quandles of cyclic type i.e., when $f=1$.

\section{Acknowledgements}

P.L. acknowledges support from FCT (Fundação para a Ciência e a Tecnologia), Portugal, through project FCT PTDC/MAT-PUR/31089/2017, "Higher Structures and Applications".

\section{References}

[1] N. Andruskiewitsch and M. Graña. From racks to pointed Hopf algebras. Adv. Math., 178(2):177-243, 2003.

[2] J. Bojarczuk and P. Lopes. Quandles at finite temperatures III. J. Knot Theory Ramifications, 14(3):275-373, 2005.

[3] E. Brieskorn. Automorphic sets and braids and singularities. Contemp. Math., 78:45$115,1988$.

[4] J. S. Carter, S. Kamada and M. Saito. Geometric interpretations of quandle homology. J. Knot Theory Ramifications, 10(3):345-386, 2001.

[5] J. S. Carter, D. Jelsovsky, S. Kamada, L. Langford and M. Saito. State-sum invariants of knotted curves and surfaces from quandle cohomology. Trans. Amer. Math. Soc., 355(10):3947-3989, 2003.

[6] J. S. Carter, S. Kamada and M. Saito. Surfaces in 4-space. Encyclopaedia of Mathematical Sciences, no. 142. Springer, Berlin, 2004.

[7] F. M. Dionísio and P. Lopes. Quandles at finite temperatures II. J. Knot Theory Ramifications, 12(8):1041-1092, 2003.

[8] P. Dehornoy. Braids and self-distributivity. Progress in Mathematics, no. 192. Birkhäuser, 2000.

[9] R. Fenn and C. Rourke. Racks and links in codimension two. J. Knot Theory Ramifications, 1(4):343-406, 1992. 
[10] C. Hayashi. Canonical forms for operation tables of finite connected quandles. Comm. Algebra, 41(9):3340-3349, 2013.

[11] D. Joyce. A classifying invariant of knots, the knot quandle. J. Pure Appl. Algebra, 23:37-65, 1982.

[12] S. Kamada, H. Tamaru and K. Wada. On classification of quandles of cyclic type. Tokyo J. Math., 39(1):157-171, 2016.

[13] L. H. Kauffman. Knots and Physics. 4th Ed., Series on Knots and Everything, no. 1. World Scientific, 2012.

[14] P. Lopes and D. Roseman. On finite racks and quandles. Comm. Algebra, 34:371-406, 2006.

[15] S. V. Matveev. Distributive groupoids in knot theory. Sb. Math., 47(1): 73-83, 1984.

[16] J. Rotman. An introduction to the theory of groups. 4th Ed., Graduate Texts in Mathematics, no. 148. Springer, 1995.

[17] L. Vendramin. Doubly transitive groups and quandles of cyclic type. J. Math. Soc. Japan, 69(3):1051-1057, 2017.

[18] T. Watanabe. On connected quandles with the profile $\{1, l, l\}$ or $\{1, l, l, l\}$. Yokohama Mathematical Journal, 61:29-39, 2015.

[19] T. Watanabe. The structure of connected quandles with the profile $\{1, l, l\}$ or $\{1, l, l, l\}$ and its inner group. J. Knot Theory Ramifications, 26 1750001, 2017.

[20] T. Watanabe. On the structure of the profile of finite connected quandles. Math. J. Okayama Univ., 61: 85-98, 2019. 